Mathematische Annalen Manuscript-Nr.

(will be inserted by hand later)

\title{
Tangent measure distributions of fractal measures
}

\author{
Peter Mörters ${ }^{1}$ and David Preiss ${ }^{2 \star}$ \\ 1 Universität Kaiserslautern, Fachbereich Mathematik, 67663 Kaiserslautern, Germany. \\ 2 University College London, Department of Mathematics, London WC1E 6BT, U.K. \\ December 9, 1996
}

Summary. Tangent measure distributions appeared as a natural tool for the description of the regularity of the local geometry of self similar sets in Euclidean spaces first in weaker versions, as in Bedford and Fisher [BeFi], and then in their full strength, as in Bandt [Ba] or Graf [G]. The results are best expressed using U. Zähle's [ZU] definition of statistical self similarity: At almost every point, the local geometry of a self similar set is, from a statistical point of view, described by a unique statistically self similar random measure.

The definition of tangent measure distributions extends naturally to arbitrary measures and dimensions. In this paper we show that for any dimension $\alpha$ and every measure on a Euclidean space, at almost every point, all $\alpha$-dimensional tangent measure distributions define statistically self similar random measures. Consequently, the local geometry of general measures is not different from the local geometry of self similar sets. The strength of this result is illustrated by pointing out how it can be used to improve or generalize recently proved relations between ordinary and average densities (e.g. [FaSp], [Ma2], or [Mö2]).

Mathematics Subject Classification (1995): 28A80, 28A75, 60G57.

\section{The result and its background}

The study of the regularity of the local behaviour of a measure $\mu$ on a Euclidean space is much simplified by the fact that, under the presence of mild a priori estimates, the weak compactness of bounded sets of measures allows one to define the measures representing the local behaviour of $\mu$ at a point $x$ as limit points of enlargements of $\mu$ about $x$. In various disguises, this idea has been used for

\footnotetext{
* Research was supported by the ARC-Programme of British Council and DAAD.
} 
a long time: Examples include Young measures (see e.g. [V]), tangent cones to currents (see [Fe, 4.3.16]), or tangent measures (see [Pr]). The applicability of this extremely valuable tool stems from the observation that taking this particular limit of measures often improves their geometric behaviour; this behaviour then can be analyzed and, by approximation, information may be obtained about the original measure. The concept of tangent measures turned out to be particularly useful for study of sets and measures of integer dimension and their rectifiability questions (see [Mat] for a description; for new results in this direction see [O2]). However, in order to investigate non-rectifiable sets and measures more refined tools seem to be necessary, as simple examples show that the set of tangent measures may be rather large and contain measures that do not have much better geometric properties than the original one.

One class of tools for the investigation of non-rectifiable measures is based on an averaging idea of Bedford and Fisher (see [BeFi]). Instead of looking directly at all limit points of the enlargements of $\mu$ about $x$ as the enlargement factor goes to infinity, we take a natural family of probability distributions on the set of positive reals whose expectations tend to infinity and study the limiting distributions of the induced family of distributions on the set of enlargements. The limiting distributions define "random tangent measures" which are called tangent measure distributions. This idea was applied by Bandt [Ba] and Graf [G] to the particular case of self similar measures. They found that self similar sets have a unique tangent measure distribution at almost all points, which they described explicitly. This technique has also been applied to statistically self similar random measures by Arbeiter and Patzschke [APa].

The fact that $\alpha$-dimensional tangent measure distributions can be defined in a natural way for all measures on Euclidean spaces and all dimensions led us to investigate to what extent the strong results on connections between rectifiability and the behaviour of tangent measures have their analogy in the fractal world. More precisely, we were looking for a converse of the results of Bandt and Graf: As the tangent measure distributions of a self similar set have been described in the work of the authors quoted above as being scaling invariant Palm measures, one would ask if self similarity of a set is equivalent to all of its tangent measure distributions being Palm measures (bearing in mind that the scaling invariance is always fulfilled).

This conjecture has turned out to be wrong. Instead we found a surprising and strong result of opposite nature: We prove here that all $\alpha$-dimensional tangent measure distributions to all measures (on Euclidean spaces of arbitrary dimension) are Palm measures and thus the local geometry of general measures is, from the statistical point of view, not different from the local geometry of self similar sets. Of course, we pay slightly for the generality of the result by allowing that the zero measure has positive probability (which should be imagined as the case when the measure has, in reality, dimension bigger than $\alpha$ ) or that it is not a probability measure (which should be imagined as the case when the measure has, in reality, dimension smaller than $\alpha$ ). We believe that this result reveals a wealth of new unexpected information about the structure of such general measures, and we illustrate this by giving several examples of its application to questions studied previously in [MöPr], [Mö2] [FaSp] and [Ma2].

We should also remark that our result is new already on the line. However, in the important case of measures on the line with positive lower and finite upper 
densities it is possible to deduce the Palm property of tangent measure distributions from very detailed information about the structure of these measures which has been obtained in the thesis [Mö1] and will appear in [Mö2].

\section{Measure theoretical definitions and preliminaries}

We first recall some notions concerning measures. If $X$ is a separable and completely metrizable topological space, short a Polish space, a non-negative Borel measure $\nu$ is termed a Radon measure if every compact subset $K$ of $X$ has finite measure and

$$
\nu(B)=\sup \{\nu(K): K \subseteq B, K \text { compact }\} \text { for every Borel set } B \subseteq X .
$$

We shall often use the facts that Radon measures $\nu$ on $X$ also fulfill (see e.g. [S, $\mathrm{I}, \S \mathrm{I} .2])$

$$
\nu(B)=\inf \{\nu(U): B \subseteq U, U \text { open }\} \text { for every Borel set } B \subseteq X
$$

and that, under the conditions imposed upon $X$, a Borel measure is Radon if and only if it is locally finite, i.e. every point has a neighbourhood of finite measure (see e.g.[S, I, $\S I I .3]$ ).

If $\nu_{k}$ are Radon measures on $X$, we say that the sequence $\left(\nu_{k}\right)$ converges vaguely to the Radon measure $\nu$ if the following two conditions hold

1. $\nu(K) \geq \lim \sup _{k \rightarrow \infty} \nu_{k}(K)$ for every compact set $K \subseteq X$, and

2. $\nu(L) \leq \liminf _{k \rightarrow \infty} \nu_{k}(L)$ for every open set $L \subseteq X$.

In the following we recall some useful descriptions of the vague convergence of Radon measures. For this purpose the following lemma, which is proved for finite measures in [T, Lemma 7.7], will turn out to be useful.

Lemma 1. Let $\left(\nu_{k}\right)$ be a sequence of Radon measures on a Polish space $X$. Then, for every $\eta>0$ and every open set $G \subseteq X$ and compact set $K \subseteq X$ with $K \subseteq G$, there are a compact set $C$ with $K \subseteq C \subseteq G$ and an open set $H$ with $K \subseteq H \subseteq G$ such that

(a) $\eta+\lim \sup _{k \rightarrow \infty} \nu_{k}(C) \geq \lim \sup _{k \rightarrow \infty} \nu_{k}(H)$ and

(b) $\eta+\liminf _{k \rightarrow \infty} \nu_{k}(C) \geq \liminf _{k \rightarrow \infty} \nu_{k}(H)$.

Proof. Let $G_{j}$ be the intersection of $G$ with the open $1 / j$-neighbourhoods of $K$. Pick strictly monotonic sequences $\left(a_{j}\right)$ increasing to $\inf _{i} \lim \sup \nu_{k}\left(G_{i}\right)$ and $\left(b_{j}\right)$ increasing to $\inf _{i} \liminf \nu_{k}\left(G_{i}\right)$. We can find a sequence $k_{1}<k_{2}<\ldots$ of indices such that $\nu_{k}\left(G_{j}\right)>b_{j}$ for all $k \geq k_{j}$ and $\nu_{l}\left(G_{j}\right)>a_{j}$ for some $k_{j} \leq l<k_{j+1}$. For every $j$ we may pick a compact set $K \subseteq C_{j} \subseteq G_{j}$ such that $\nu_{k}\left(\bar{C}_{j}\right) \geq b_{j}$ for all $k_{j} \leq k<k_{j+1}$ and $\nu_{l}\left(C_{j}\right) \geq a_{j}$ for some $k_{j} \leq l<k_{j+1}$. Then the set $C=\bigcup C_{j}$ is compact (see e.g. [T, Lemma 7.5.]) and fulfills $\liminf \nu_{k}(C) \geq \liminf b_{j}$ and $\lim \sup \nu_{k}(C) \geq \lim \sup a_{j}$. Picking $j$ large enough such that $\bar{H}=G_{j}$ fulfills $\limsup \nu_{k}(H) \leq \inf _{i} \limsup \nu_{k}\left(G_{i}\right)+\eta$ and $\liminf \nu_{k}(H) \leq \inf _{i} \liminf \nu_{k}\left(G_{i}\right)+\eta$ finishes the proof. 
Lemma 2. Let $\left(\nu_{k}\right)$ be a sequence of Radon measures on a Polish space $X$ converging vaguely to the Radon measure $\nu$.

(a) For every compact $K \subseteq X$ and every $\eta>0$, there is an open set $H \supseteq K$ such that $\lim \sup _{k \rightarrow \infty} \nu_{k}(H)<\nu(K)+\eta$.

(b) For every open set $G \subseteq X$ with $\nu(G)<\infty$ and every $\eta>0$, there is a compact set $C \subseteq G$ such that $\liminf _{k \rightarrow \infty} \nu_{k}(C)>\nu(G)-\eta$.

Proof. To prove (a) we pick an open set $G \supseteq K$ such that $\nu(G \backslash K)<\eta / 2$. By Lemma 1(a) we can find a compact set $C \subseteq G$ and an open set $H \supseteq K$ with $\lim \sup \nu_{k}(H) \leq \eta / 2+\lim \sup \nu_{k}(C)$ and, as $\overline{\lim \sup } \nu_{k}(C) \leq \nu(C)<\nu \overline{(K)}+\eta / 2$, this proves (a). To prove (b) we pick a compact set $K \subseteq G$ with $\nu(G \backslash K)<\eta / 2$ and use Lemma 1(b) to find a compact set $C \subseteq G$ and an open set $H \supseteq K$ with $\eta / 2+\liminf \nu_{k}(C) \geq \liminf \nu_{k}(H)$. As $\liminf \nu_{k}(H) \geq \nu(H)>\nu(G)-\eta / 2$, (b) is proved.

Lemma 3. A sequence $\left(\nu_{k}\right)$ of Radon measures on a Polish space $X$ converges vaguely to a Radon measure $\nu$ if and only if for every compact set $K \subseteq X$ and every $\eta>0$ there is an open set $K \subseteq L \subseteq X$ such that

$$
\limsup _{k \rightarrow \infty}\left|\int \varphi(x) d \nu_{k}(x)-\int \varphi(x) d \nu(x)\right|<\eta
$$

for every continuous function $\varphi: X \rightarrow[0,1]$ such that $\varphi(x)=0$ for $x \notin L$.

Proof. An easy approximation shows that the given condition implies that $\left(\nu_{k}\right)$ converges vaguely to $\nu$. On the other hand, if the convergence is assumed, $\eta>0$ and $K \subseteq X$ is compact, we can use Lemma 2(a) and (b) to first find an open set $L \supseteq K$ such that $\lim \sup \nu_{k}(L)<\nu(K)+\eta / 2$ and then a compact set $C$ with $\bar{K} \subseteq C \subseteq L$ with $\lim \sup \nu_{k}(L \backslash C)<\eta$. Observe that $\sup \nu_{k}(C)<\infty$. If $\varphi$ is given as in the lemma, we calculate, using Fubini's Theorem and Fatou's Lemma,

$$
\begin{aligned}
\int \varphi d \nu & \geq \int_{C} \varphi d \nu=\int_{0}^{1} \nu(\{x \in C: \varphi(x) \geq t\}) d t \\
& \geq \int_{0}^{1} \limsup _{k \rightarrow \infty} \nu_{k}(\{x \in C: \varphi(x) \geq t\}) d t \\
& \geq \limsup _{k \rightarrow \infty} \int_{0}^{1} \nu_{k}(\{x \in C: \varphi(x) \geq t\}) d t \\
& =\limsup _{k \rightarrow \infty} \int_{C} \varphi d \nu_{k} \geq \limsup _{k \rightarrow \infty} \int \varphi d \nu_{k}-\eta,
\end{aligned}
$$

and, similarly, we get

$$
\begin{aligned}
\int \varphi d \nu & =\int_{0}^{1} \nu(\{x \in L: \varphi(x)>t\}) d t \\
& \leq \int_{0}^{1} \liminf _{k \rightarrow \infty} \nu_{k}(\{x \in L: \varphi(x)>t\}) d t \\
& \leq \liminf _{k \rightarrow \infty} \int_{0}^{1} \nu_{k}(\{x \in L: \varphi(x)>t\}) d t=\liminf _{k \rightarrow \infty} \int \varphi d \nu_{k}
\end{aligned}
$$


which together implies the condition given in the lemma.

As an important consequence of the lemma we note that the vague limit of a sequence $\left(\nu_{k}\right)$, if it exists, is uniquely determined. Although we have not defined a topology inducing the vague convergence (see [T, Chap. 7] for problems related to this), the notion of relative sequential compactness with respect to the vague convergence is naturally defined for a set of Radon measures.

Lemma 4. A set $M$ of Radon measures on a Polish space $X$ is relatively sequentially compact with respect to the vague convergence if and only if $\sup \{\nu(K)$ : $\nu \in M\}<\infty$ for every compact set $K \subseteq X$.

Proof. If there is a sequence $\left(\nu_{k}\right)$ in $M$ such that, for some compact $K$, the sequence $\nu_{k}(K)$ tends to $\infty$, this sequence clearly has no convergent subsequence and $M$ cannot be relatively sequentially compact. On the other hand suppose $\left(\nu_{k}\right)$ is a sequence in $M$ and $\left(\nu_{k}(K)\right)$ is bounded for every compact $K$. We have to find a convergent subsequence. Using Lemma 1(a) we can find a sequence $\left(G_{j}\right)$ of open sets increasing to $X$ with $\sup _{k} \nu_{k}\left(G_{j}\right)<\infty$. As any family of finite measures with bounded mass has a vaguely convergent subsequence (see e.g.[T, Theorem 7.8]), we can pick a subsequence $\left(\mu_{k}\right)$ of $\left(\nu_{k}\right)$ such that, for every $j$, the sequence $1_{G_{j}} \mu_{k}$ converges, say to $\mu^{(j)}$. For every compact set $K$ there is an index $n$ such that $K \subseteq G_{n}$ and therefore $\left.\mu^{(i)}\right|_{K}=\left.\mu^{(j)}\right|_{K}$ for all $i, j \geq n$. Using this, it is easy to see that we can define a Radon measure $\mu$ by $\mu(B)=\sup \mu^{(n)}(K)$, where the supremum extends over all compact sets $K \subseteq B$ and $n$ is chosen according to $K$. It is then straightforward to check that $\bar{\mu}$ is indeed the limit of the sequence $\left(\mu_{j}\right)$.

Lemma 5. Suppose that $\mu_{k}$ and $\nu_{k}$ are Radon measures on a Polish space $X$ such that $\left(\mu_{k}\right)$ converges vaguely to $\mu_{0}$ and $\left(\nu_{k}\right)$ converges vaguely to $\nu_{0}$. Suppose further that for every $\eta>0$ and for every pair $K \subseteq L$ of subsets of $X$ such that $K$ is compact and $L$ is open there is an open set $\bar{K} \subseteq U \subseteq L$ such that

$$
\liminf _{k \rightarrow \infty} \mu_{k}(U) \leq \eta+\limsup _{k \rightarrow \infty} \nu_{k}(L) \text {. }
$$

Then $\mu_{0} \leq \nu_{0}$

Proof. If $K \subseteq X$ is compact and $\eta>0$, we use Lemma 3 to find an open set $L \supseteq K$ such that

$$
\limsup _{k \rightarrow \infty}\left|\int \varphi(x) d \mu_{k}(x)-\int \varphi(x) d \mu_{0}(x)\right|<\eta
$$

and

$$
\limsup _{k \rightarrow \infty}\left|\int \varphi(x) d \nu_{k}(x)-\int \varphi(x) d \nu_{0}(x)\right|<\eta
$$

for every continuous function $\varphi: X \rightarrow[0,1]$ such that $\varphi(x)=0$ for $x \notin L$. Let $L \supseteq L_{0} \supseteq K$ be an open set such that $\nu_{0}\left(L_{0} \backslash K\right)<\eta$ and let $L_{0} \supseteq L_{1} \supseteq K$ be an open set whose closure is contained in $L_{0}$. Using the assumption of the lemma, we find an open set $L_{1} \supseteq U \supseteq K$ such that

$$
\liminf _{k \rightarrow \infty} \mu_{k}(U) \leq \eta+\limsup _{k \rightarrow \infty} \nu_{k}\left(L_{1}\right)
$$


Let $\varphi: X \rightarrow[0,1]$ be a continuous function such that $\varphi(x)=1$ for $x \in K$ and $\varphi(x)=0$ for $x \notin U$ and let $\psi: X \rightarrow[0,1]$ be a continuous function such that $\psi(x)=1$ for $x \in L_{1}$ and $\psi(x)=0$ for $x \notin L_{0}$. Then

$$
\begin{aligned}
\mu_{0}(K) & \leq \int \varphi(x) d \mu_{0}(x) \leq \eta+\liminf _{k \rightarrow \infty} \int \varphi(x) d \mu_{k}(x) \leq \eta+\liminf _{k \rightarrow \infty} \mu_{k}(U) \\
& \leq 2 \eta+\limsup _{k \rightarrow \infty} \nu_{k}\left(L_{1}\right) \leq 2 \eta+\limsup _{k \rightarrow \infty} \int \psi(x) d \nu_{k}(x) \\
& \leq 3 \eta+\int \psi(x) d \nu_{0}(x) \leq 3 \eta+\nu_{0}\left(L_{0}\right) \leq 4 \eta+\nu_{0}(K) .
\end{aligned}
$$

Since $\eta>0$ and the compact set $K$ are arbitrary, this shows that $\mu_{0} \leq \nu_{0}$.

A sequence $\left(\nu_{k}\right)$ of Radon measures with $\lim \sup _{k \rightarrow \infty} \nu_{k}(X)<\infty$ is said to converge weakly to the Radon measure $\nu$ if $\int \varphi d \nu_{k} \rightarrow \int \varphi d \nu$ for every bounded continuous function $\varphi$ on $X$.

Lemma 6. Let $\nu_{k}$ be Radon measures on a Polish space $X$ and suppose that $\limsup _{k \rightarrow \infty} \nu_{k}(X)<\infty$ and $\left(\nu_{k}\right)$ converges vaguely to a Radon measure $\nu$. Then the following statements are equivalent:

(a) $\left(\nu_{k}\right)$ converges weakly to $\nu$.

(b) $\nu(X) \geq \lim \sup _{k \rightarrow \infty} \nu_{k}(X)$.

(c) For every $\eta>0$ there is $K \subseteq X$, compact, such that $\lim \sup \nu_{k}(X \backslash K) \leq \eta$.

Proof. As the function $1_{X}$ is continuous and bounded, (a) implies (b). If (b) holds, then $\nu(A) \geq \limsup \operatorname{su}_{k \rightarrow \infty} \nu_{k}(A)$ for every closed set $A \subseteq X$ and we can conclude the weak convergence by means of Fubini's Theorem as exercised in the proof of Lemma 3. Finally, the equivalence of (a) and (c) follows from Prohorov's Theorem (see [S, App. $\S 3$, Th. 3 and 4]).

Of course, if the Polish space $X$ is compact, then Radon measures are finite and weak and vague convergence coincide. More generally, if $X$ is a locally compact Polish space, then $\nu_{k}$ converges vaguely to $\nu$ if and only if $\int \varphi d \nu_{k} \rightarrow \int \varphi d \nu$ for every continuous function $\varphi$ on $X$ with compact support. Then the vague convergence of Radon measures is induced by the vague topology, the smallest topology on the set of Radon measures that makes the mappings $\mu \mapsto \int \varphi d \mu$ continuous for all continuous $\varphi$ with compact support. The space $\mathcal{M}(X)$ of Radon measures with this topology is again a Polish space.

Lemma 7. Suppose that $X$ is a locally compact Polish space and that $P_{k}$ are Radon measures on $\mathcal{M}(X)$ vaguely converging to $P_{0}$. Let $\Lambda_{k}$ be Borel measures on $\mathcal{M}(X) \times X$ defined by

$$
\Lambda_{k}(E)=\iint 1_{E}(\nu, y) d \nu(y) d P_{k}(\nu) .
$$

Then $\Lambda_{k}$ are Radon measures converging vaguely to $\Lambda_{0}$. 
Proof. If $K \subseteq \mathcal{M}(X) \times X$ is compact, let $K_{1} \subseteq \mathcal{M}(X)$ and $K_{2} \subseteq X$ be compact sets such that $K \subseteq K_{1} \times K_{2}$ and let $L_{2} \supseteq \bar{K}_{2}$ be an open set contained in a compact set $K_{3} \subseteq X$. Then there is $0<c<\infty$ such that $\nu\left(K_{3}\right)<c$ for all $\nu \in K_{1}$. Given any $\eta>0$, we use Lemma 3 to find an open set $L_{1} \supseteq K_{1}$ such that

$$
\limsup _{k \rightarrow \infty}\left|\int \varphi(\nu) d P_{k}(\nu)-\int \varphi(\nu) d P_{0}(\nu)\right|<\eta / c
$$

for every continuous function $\varphi: \mathcal{M}(X) \rightarrow[0,1]$ such that $\varphi(\nu)=0$ for $\nu \notin L_{1}$; intersecting $L_{1}$ with the open set $\left\{\nu: \nu\left(K_{3}\right)<c\right\}$, if necessary, we may also assume that $\nu\left(L_{2}\right)<c$ for all $\nu \in L_{1}$. As a byproduct we note that $K_{1} \times L_{2}$ contains $K$ and $\Lambda_{k}\left(K_{1} \times L_{2}\right)<\infty$, so that $\Lambda_{k}$ are Radon measures. Denote $L=L_{1} \times L_{2}$. If $\psi: \mathcal{M}(X) \times X \rightarrow[0,1]$ is a continuous function such that $\psi(\nu, y)=0$ if $(\nu, y) \notin L$, we note that the function $\varphi(\nu)=\frac{1}{c} \int \psi(\nu, y) d \nu(y)$ is non-negative, continuous, bounded by one, and satisfies $\varphi(\nu)=0$ for $\nu \notin L_{1}$. Thus (1) implies that

$$
\limsup _{k \rightarrow \infty}\left|\int \psi(\nu, y) d \Lambda_{k}(\nu, y)-\int \psi(\nu, y) d \Lambda_{0}(\nu, y)\right|<\eta,
$$

which, according to Lemma 3 , implies that $\left(\Lambda_{k}\right)$ vaguely converges to $\Lambda_{0}$.

In the case $X=\mathbf{R}^{d}$ the set $\mathcal{M}\left(\mathbf{R}^{d}\right)$ of all Radon measures on $\mathbf{R}^{d}$ equipped with the vague topology is a Polish space and we observe, as a consequence of Lemma 4 , that a subset $M$ of $\mathcal{M}\left(\mathbf{R}^{d}\right)$ is relatively compact if and only if for each $n=1,2, \ldots$ there is $c(n) \in(0, \infty)$ such that $\mu U(0, n) \leq c(n)$ for each $\mu \in M$, where $U(x, r)$ denotes the open Euclidean ball of radius $r$ centred in $x$.

Finite Radon measures on the Polish space $\mathcal{M}\left(\mathbf{R}^{d}\right)$ will be termed measure distributions on $\mathbf{R}^{d}$; depending on the context we may also call them random measures. The measure distributions we are interested in will always be subprobability measures and often, but not always, they will be probability measures.

\section{Tangent measure distributions and their basic properties}

The main geometric measure theoretical notions describing the $\alpha$-dimensional part of the behaviour of a Radon measure $\mu$ on $\mathbf{R}^{d}$ about a point $x \in \mathbf{R}^{d}$ are introduced in the following way. We define the family of measures $\left(\mu_{x, r}\right)_{r>0} \subseteq$ $\mathcal{M}\left(\mathbf{R}^{d}\right)$, the enlargements of $\mu$ about $x$, by $\mu_{x, r}(A)=\mu(x+r A)$ for all Borel sets $A \subseteq \mathbf{R}^{d}$ and probability distributions $P_{\delta}^{x}$ on $\mathcal{M}\left(\mathbf{R}^{d}\right)$ by

$$
P_{\delta}^{x}(M)=(|\log \delta|)^{-1} \int_{\delta}^{1} 1_{M}\left(\frac{\mu_{x, r}}{r^{\alpha}}\right) \frac{d r}{r} \text { for Borel sets } M \subseteq \mathcal{M}\left(\mathbf{R}^{d}\right) .
$$

$\mathcal{P}^{\alpha}(\mu, x)$ is defined as the set of all limit points of $\left(P_{\delta}^{x}\right)_{\delta>0}$ as $\delta \downarrow 0$ in the vague convergence. The elements of $\mathcal{P}^{\alpha}(\mu, x)$ are the $\alpha$-dimensional tangent measure distributions of $\mu$ at $x$. We observe that $\mathcal{P}^{\alpha}(\mu, x) \neq \emptyset$; indeed, Lemma 4 shows that for every sequence $\delta_{k} \downarrow 0$ the sequence $P_{\delta_{k}}^{x}$ has a vaguely convergent subsequence. 
This approach also allows us to study the local geometry of sets $E \subseteq \mathbf{R}^{d}$ by means of natural measures on the set, like for example Hausdorff measures.

We also point out that no a priori relation between $\alpha, \mu$ and $x$ has been assumed; thus, for example, tangent measure distributions of the one dimensional Lebesgue measure $\mathcal{L}$ in $\mathbf{R}$ are:

1. If $\alpha<1$ then the unique $\alpha$-dimensional tangent measure distribution of $\mathcal{L}$ at every $x \in \mathbf{R}$ is the Dirac measure concentrated at the zero measure.

2. If $\alpha=1$ then the unique $\alpha$-dimensional tangent measure distribution of $\mathcal{L}$ at every $x \in \mathbf{R}$ is the Dirac measure concentrated at $\mathcal{L}$.

3 . If $\alpha>1$ then the unique $\alpha$-dimensional tangent measure distribution of $\mathcal{L}$ at every $x \in \mathbf{R}$ is the zero measure distribution.

Several close concepts have been used to describe the behaviour of a Radon measure $\mu$ on $\mathbf{R}^{d}$ about a point. The most classical are the lower and upper $\alpha$-dimensional densities of $\mu$ at $x$ defined by

$$
\liminf _{r \downarrow 0} \frac{\mu(U(x, r))}{r^{\alpha}} \quad \text { and } \quad \limsup _{r \downarrow 0} \frac{\mu(U(x, r))}{r^{\alpha}},
$$

respectively, where $U(x, r)$ denotes the open Euclidean ball of radius $r$ with centre at $x$. More recent is the notion of lower and upper average, or order two, $\alpha$-dimensional densities of $\mu$ at $x$ defined by

$$
\liminf _{\delta \downarrow 0}(|\log \delta|)^{-1} \int_{\delta}^{1} \frac{\mu(U(x, r))}{r^{\alpha}} \frac{d r}{r} \text { and } \limsup _{\delta \downarrow 0}(|\log \delta|)^{-1} \int_{\delta}^{1} \frac{\mu(U(x, r))}{r^{\alpha}} \frac{d r}{r}
$$

respectively. Finally, the $\alpha$-dimensional tangent measures of $\mu$ at $x$ are defined as elements of the set

$$
\operatorname{Tan}_{\alpha}(\mu, x)=\left\{\nu=\lim _{n \rightarrow \infty} \frac{\mu_{x, r_{n}}}{r_{n}^{\alpha}} \text { in the vague topology for some } r_{n} \downarrow 0\right\} .
$$

Again, no relation between $\alpha, \mu$ and $x$ has been assumed; thus, for example, $\operatorname{Tan}_{2}(\mathcal{L}, x)=\emptyset$ for every $x \in \mathbf{R}$. Note also that $\alpha$-dimensional tangent measures may form a proper subset of the set of tangent measures considered in $[\mathrm{Pr}]$.

We shall give some statements concerning compactness, localization and scaling invariance properties of tangent measure distributions.

Proposition 1. Suppose that $\mu \in \mathcal{M}\left(\mathbf{R}^{d}\right)$ and $\alpha \geq 0$.

(a) For every $x \in \mathbf{R}^{d}$, and for every sequence $\delta_{k} \downarrow 0$ the sequence $P_{\delta_{k}}^{x}$ has a subsequence vaguely convergent to a distribution $P$ concentrated on the set $\operatorname{Tan}_{\alpha}(\mu, x)$. Hence all $P \in \mathcal{P}^{\alpha}(\mu, x)$ are concentrated on $\operatorname{Tan}_{\alpha}(\mu, x)$.

(b) If $\mu$ has finite upper average $\alpha$-dimensional density at $x$, then for every sequence $\delta_{k} \downarrow 0$ the sequence $P_{\delta_{k}}^{x}$ has a weakly convergent subsequence. In particular, all $P \in \mathcal{P}^{\alpha}(\mu, x)$ are probability measures and the set $\mathcal{P}^{\alpha}(\mu, x)$ is compact in the weak topology.

(c) If $\mu$ has positive lower $\alpha$-dimensional density at $x$, then $P(\{\phi\})=0$ for every $P \in \mathcal{P}^{\alpha}(\mu, x)$. (Here $\phi$ denotes the zero measure.) 
Proof. The existence of a vaguely convergent subsequence follows from Lemma 4. If the sequence $P_{\delta_{k}}^{x}$ vaguely converges to $P$ and $P\left(\mathcal{M}\left(\mathbf{R}^{d}\right) \backslash \operatorname{Tan}_{\alpha}(\mu, x)\right)>0$, we find a compact set $K \subset \mathcal{M}\left(\mathbf{R}^{d}\right) \backslash \operatorname{Tan}_{\alpha}(\mu, x)$ of positive $P$ measure and use that $\operatorname{Tan}_{\alpha}(\mu, x)$ is closed to find an open set $K \subseteq L$ whose closure does not meet $\operatorname{Tan}_{\alpha}(\mu, x)$. Since $K$ is compact, for every $n \equiv 1,2, \ldots$ there is a constant $c(n) \in(0, \infty)$ such that $\nu \overline{U(0, n)}<c(n)$ for every $\nu \in K$. Then the sets

$$
L_{n}=\{\nu \in L: \nu \overline{U(0,1)}<c(1), \ldots, \nu \overline{U(0, n)}<c(n)\}
$$

are open and contain $K$; so $P\left(L_{n}\right) \geq P(K)$ and we infer that for each $n$ there is $\delta<n^{-2 / P(K)}$ such that $P_{\delta}^{x}\left(L_{n}\right)>P(K) / 2$. Noting that

$$
(|\log \delta|)^{-1} \int_{1 / n}^{1} 1_{L_{n}}\left(\frac{\mu_{x, r}}{r^{\alpha}}\right) \frac{d r}{r} \leq(|\log \delta|)^{-1} \log n<P(K) / 2,
$$

we conclude that there is $0<r_{n}<1 / n$ such that $\frac{\mu_{x, r_{n}}}{r_{n}^{\alpha}} \in L_{n}$. Now the sequence $\frac{\mu_{x, r_{n}}}{r_{n}^{\alpha}}$ has a convergent subsequence; but its limit has to belong to $\operatorname{Tan}_{\alpha}(\mu, x)$ as well as to the closure of the set $L$, which is disjoint from it. This contradiction finishes the proof of (a).

If $\mu$ has finite upper average $\alpha$-dimensional density at $x$, we infer from

$$
(|\log \delta|)^{-1} \int_{\delta}^{1} \frac{\mu(U(x, n r))}{r^{\alpha}} \frac{d r}{r}=n^{\alpha}(|\log \delta|)^{-1} \int_{n \delta}^{n} \frac{\mu(U(x, r))}{r^{\alpha}} \frac{d r}{r}
$$

that the numbers

$$
D_{n}=\sup \left\{(|\log \delta|)^{-1} \int_{\delta}^{1} \frac{\mu(U(x, n r))}{r^{\alpha}} \frac{d r}{r}: 0<\delta<1 / 2\right\}
$$

are finite. Thus for any $\eta>0$ we may choose $c(n) \in(0, \infty)$ such that $\sum_{n=1}^{\infty} \frac{D_{n}}{c(n)}<\eta$ and observe that the set

$$
K=\left\{\nu \in \mathcal{M}\left(\mathbf{R}^{d}\right): \nu U(0, n) \leq c(n) \text { for } n=1,2, \ldots\right\}
$$

is compact and that by Chebyshev's inequality $P_{\delta}^{x}\left(\mathbf{R}^{d} \backslash K\right)<\eta$. Hence the existence of a weakly convergent subsequence and the compactness statement follows from Lemmas 4 and 6 and the fact that $\mathcal{P}^{\alpha}(\mu, x)$ consist of probability measures follows from Lemma 6 .

Finally, if $\mu$ has positive lower $\alpha$-dimensional density at $x$, then $\operatorname{Tan}_{\alpha}(\mu, x)$ does not contain the zero measure. Hence (c) follows from (a).

Remark. Observe that by Proposition 1, whenever $\mu$ has positive lower and finite upper $\alpha$-dimensional densities at $x$, all tangent measure distributions $P \in$ $\mathcal{P}^{\alpha}(\mu, x)$ are nontrivial in the sense that $P\left(\mathcal{M}\left(\mathbf{R}^{d}\right) \backslash\{\phi\}\right)=1$.

Using Proposition 1 we can find the $\alpha$-dimensional tangent measure distributions in several trivial situations.

Proposition 2. For every measure $\mu \in \mathcal{M}\left(\mathbf{R}^{d}\right)$, at $\mu$-almost every $x \in \mathbf{R}^{d}$, 
(a) the only 0-dimensional tangent measure distribution is given by the Dirac measure concentrated at the $\mu(\{x\})$ multiple of the Dirac measure concentrated at the origin (in fact, this holds at every $x$ ),

(b) the only d-dimensional tangent measure distribution is given either by the zero measure or by the Dirac measure concentrated at a multiple of Lebesgue measure on $\mathbf{R}^{d}$ depending on whether $\limsup _{r \rightarrow 0} \mu U(x, r) / \mathcal{L}^{d} U(x, r)$ is infinite or finite (and, in the latter case, this limit defines the multiple),

(c) if $\alpha>d$, then the only $\alpha$-dimensional tangent measure distribution is the zero measure.

If $\mu$ is $\alpha$-rectifiable then, at $\mu$-almost every $x \in \mathbf{R}^{d}$,

(d) the $\alpha$-dimensional tangent measure distribution at $x$ is unique and given by a Dirac measure concentrated at the only tangent measure at this point, which is a constant multiple of Hausdorff measure on the approximate tangent space at the point.

Proof. This is immediate from Proposition 1 and the structure of the respective sets of $\alpha$-dimensional tangent measures (see [Mat, Chap. 14-16]).

Remark. Proposition 2(d) shows that tangent measure distributions are a natural generalization of the classical concept of a tangent space.

Lemma 8. Suppose that $\mu, \nu \in \mathcal{M}\left(\mathbf{R}^{d}\right)$ are Radon measures, $\alpha \geq 0$ and $x \in \mathbf{R}^{d}$ is such that

$$
\lim _{r \downarrow 0} \frac{\sup \{|\mu(E)-\nu(E)|: E \subseteq U(x, r)\}}{(\mu+\nu)(U(x, r))}=0
$$

and that $\delta_{k} \downarrow 0$. Then, if for either of $\mu$ or $\nu$ the sequence $P_{\delta_{k}}^{x}$ converges vaguely, then both sequences converge and they have the same limit.

Proof. Denote by $P_{k}$ and $Q_{k}$ the sequence $P_{\delta_{k}}^{x}$ with respect to $\mu$, respectively $\nu$. Because of the possibility of passing to convergent subsequences, it suffices to assume that $P_{k}$ and $Q_{k}$ converge vaguely to, say $P$ and $Q$, respectively. If $\eta>0$ and $L \subseteq \mathcal{M}\left(\mathbf{R}^{d}\right)$ is open, we use Lemma 2(b) to find a compact set $K \subseteq L$ such that $\lim \sup _{k \rightarrow \infty} P_{k}(K)>P(L)-\eta$. Since $L$ is a vague neighbourhood of the compact set $K$, there is a positive integer $m$ such that, with a suitable $0<\sigma<1, L$ contains every measure $\gamma$ which satisfies

$$
\sup \{|\gamma(E)-\beta(E)|: E \subseteq U(0, m)\} \leq \sigma \text { for some } \beta \in K .
$$

Let $c(n) \in(1, \infty)$ be such that $\beta U(0, n)<c(n)$ for all $\beta \in K$ and let $0<R<1$ be such that

$$
\frac{\sup \{|\mu(E)-\nu(E)|: E \subseteq U(x, r)\}}{(\mu+\nu)(U(x, r))}<\sigma /(3 c(m))
$$

for $0<r<R$; using this with $E=U(x, r)$, we infer that $\nu U(x, r) \leq 2 \mu U(x, r)$ and, consequently,

$$
\frac{\sup \{|\mu(E)-\nu(E)|: E \subseteq U(x, r)\}}{\mu U(x, r)}<\sigma / c(m)
$$


for $0<r<R$ and $E \subseteq U(x, r)$.

Let $K_{0}$ be the set of all measures $\gamma$ which have the property (2) and, in addition, satisfy

$$
\gamma U(0, n) \leq 2 c(n)+(n / R)^{\alpha} \nu U(x, n) \text { for every } n \geq m .
$$

Then $K \subseteq K_{0} \subseteq L$ and $K_{0}$ is compact. Moreover, if $0<r<R / m$ and $\frac{\mu_{x, r}}{r^{\alpha}} \in K$, we show that $\frac{\overline{\nu, r} r}{r^{\alpha}} \in K_{0}$. Indeed, if $n r \geq R$, then

$$
\frac{\nu_{x, r}}{r^{\alpha}}(U(0, n)) \leq \frac{\nu U(x, n)}{r^{\alpha}} \leq(n / R)^{\alpha} \nu U(x, n),
$$

if $n r<R$, then

$$
\frac{\nu_{x, r}}{r^{\alpha}}(U(0, n)) \leq \frac{\nu U(x, n r)}{\mu U(x, n r)} \frac{\mu_{x, r}}{r^{\alpha}}(U(0, n)) \leq 2 c(n)
$$

and, finally,

$$
\begin{aligned}
& \sup \left\{\left|\frac{\mu_{x, r}}{r^{\alpha}}(E)-\frac{\nu_{x, r}}{r^{\alpha}}(E)\right|: E \subseteq U(0, m)\right\} \\
& \quad \leq \frac{\sup \{|\mu(E)-\nu(E)|: E \subseteq U(x, m r)\}}{\mu U(x, m r)} \frac{\mu_{x, r}}{r^{\alpha}}(U(0, m))<\sigma .
\end{aligned}
$$

Consequently, $Q(L) \geq Q\left(K_{0}\right) \geq \lim \sup _{k \rightarrow \infty} Q_{k}\left(K_{0}\right) \geq \lim \sup _{k \rightarrow \infty} P_{k}(K)>$ $P(L)-\eta$, which, as $\eta>0$ is arbitrary, implies that $Q(L) \geq P(L)$ for every open set $L \subseteq \mathcal{M}\left(\mathbf{R}^{d}\right)$. Exchanging the roles of $P$ and $Q$, we finally conclude that $P=Q$.

Proposition 3. Suppose that $\alpha \geq 0$ and that $E \subseteq \mathbf{R}^{d}$ is $\mu$-measurable. Then the restriction of $\mu$ to $E$, which is the Radon measure $\left.\mu\right|_{E}$ on $\mathbf{R}^{d}$ defined by $\left.\mu\right|_{E}(A)=\mu(E \cap A)$, fulfills $\mathcal{P}^{\alpha}(\mu, x)=\mathcal{P}^{\alpha}\left(\left.\mu\right|_{E}, x\right)$ for $\mu$-almost every $x \in E$.

Proof. For $\mu$-almost every $x \in E$ we infer from the Besicovitch-Morse Density Theorem (see [Fe, 2.9.11]) that

$$
\lim _{r \downarrow 0} \frac{\mu(B(x, r) \backslash E)}{\mu(B(x, r))}=0 .
$$

For any such $x \in E$ the required statement holds because the measure $\mu$ and $\nu=\left.\mu\right|_{E}$ satisfy the assumptions of Lemma 8 .

Remark. Similarly, Lemma 8 may be used to describe tangent measure distributions of a measure absolutely continuous with respect to $\mu$ : If the RadonNikodym derivative of $\nu$ with respect to $\mu$ is $f$, and if $x$ is a Lebesgue point of $f$, i.e. $\lim _{r \rightarrow 0} \int_{U(x, r)}|f(x)-f(y)| d \mu(y) / \mu U(x, r)=0$, we can first transform tangent measure distributions of $\mu$ to its constant multiple by $f(x)$, and then infer from Lemma 8 that these are precisely the tangent measure distributions of $\nu$ at $x$. Since $\mu$-almost every point is a Lebesgue point of $f$, this applies in $\mu$, hence $\nu$, almost every point of $\mathbf{R}^{d}$. 
Proposition 4. For every $\lambda>0$ we define the rescaling operator $S_{\lambda}^{\alpha}: \mathcal{M}\left(\mathbf{R}^{d}\right) \rightarrow$ $\mathcal{M}\left(\mathbf{R}^{d}\right)$ by $S_{\lambda}^{\alpha} \nu(E)=\left(1 / \lambda^{\alpha}\right) \cdot \nu(\lambda E)$. For all $x \in \mathbf{R}^{d}$ and $P \in \mathcal{P}^{\alpha}(\mu, x)$ we have

$$
P=P \circ\left(S_{\lambda}^{\alpha}\right)^{-1} \text { for all } \lambda>0 .
$$

Proof. Let $\lambda>0$ and observe that $S_{\lambda}^{\alpha}$ is a homeomorphism of $\mathcal{M}\left(\mathbf{R}^{d}\right)$ onto itself, hence it preserves compact and open subsets of $\mathcal{M}\left(\mathbf{R}^{d}\right)$. Suppose that $\delta_{k} \downarrow 0$ and that $P$ is the vague limit of $P_{\delta_{k}}^{x}$. For every compact set $K \subseteq \mathcal{M}\left(\mathbf{R}^{d}\right)$ and every open set $L \subseteq \mathcal{M}\left(\mathbf{R}^{d}\right)$ we calculate

$$
\begin{aligned}
\left(P \circ\left(S_{\lambda}^{\alpha}\right)^{-1}\right)(K) & =P\left(\left(S_{\lambda}^{\alpha}\right)^{-1}(K)\right) \\
& \geq \limsup _{k \rightarrow \infty}\left(\left|\log \delta_{k}\right|\right)^{-1} \int_{\delta_{k}}^{1} 1_{\left(S_{\lambda}^{\alpha}\right)^{-1}(K)}\left(\frac{\mu_{x, r}}{r^{\alpha}}\right) \frac{d r}{r} \\
& =\limsup _{k \rightarrow \infty}\left(\left|\log \delta_{k}\right|\right)^{-1} \int_{\delta_{k}}^{1} 1_{K}\left(\frac{\mu_{x, \lambda r}}{(\lambda r)^{\alpha}}\right) \frac{d r}{r} \\
& =\limsup _{k \rightarrow \infty}\left(\left|\log \delta_{k}\right|\right)^{-1} \int_{\delta_{k} / \lambda}^{1 / \lambda} 1_{K}\left(\frac{\mu_{x, \lambda r}}{(\lambda r)^{\alpha}}\right) \frac{d r}{r} \\
& =\limsup _{k \rightarrow \infty}\left(\left|\log \delta_{k}\right|\right)^{-1} \int_{\delta_{k}}^{1} 1_{K}\left(\frac{\mu_{x, r}}{r^{\alpha}}\right) \frac{d r}{r} \\
& =\limsup _{k \rightarrow \infty} P_{\delta_{k}}^{x}(K)
\end{aligned}
$$

and, similarly, $\left(P \circ\left(S_{\lambda}^{\alpha}\right)^{-1}\right)(L) \leq \liminf _{k \rightarrow \infty} P_{\delta_{k}}^{x}(L)$. Thus $P_{\delta_{k}}^{x}$ converges vaguely to $P \circ\left(S_{\lambda}^{\alpha}\right)^{-1}$, which, because of the unique determination of the limit, proves that $P=P \circ\left(S_{\lambda}^{\alpha}\right)^{-1}$.

\section{The main result}

While the scaling invariance of tangent measure distributions is easy, it is a more interesting and difficult task to formulate and prove their shift-invariance property. For every $u \in \mathbf{R}^{d}$ we define the shift operator $T^{u}: \mathcal{M}\left(\mathbf{R}^{d}\right) \rightarrow \mathcal{M}\left(\mathbf{R}^{d}\right)$ by $T^{u} \nu(E)=\nu(u+E)$. A shift-invariance principle for tangent measure distributions is given in the following theorem.

Theorem 1. Let $\alpha \geq 0$ and let $\mu \in \mathcal{M}\left(\mathbf{R}^{d}\right)$ be any measure. Then at $\mu$-almost all points $x$ every $\alpha$-dimensional tangent measure distribution $P \in \mathcal{P}^{\alpha}(\mu, x)$ fulfills

$$
\iint G(\nu, u) d \nu(u) d P(\nu)=\iint G\left(T^{u} \nu,-u\right) d \nu(u) d P(\nu)
$$

for all Borel functions $G: \mathcal{M}\left(\mathbf{R}^{d}\right) \times \mathbf{R}^{d} \longrightarrow[0, \infty)$.

Remark. Because of the simple description of tangent measure distributions of rectifiable measures (see Proposition 2(d)), the formula is easy whenever $\mu$ is rectifiable. In particular it holds trivially in the special cases $\alpha=0$ and $\alpha \geq d$. 
Since the formulation of the translational invariance property may not be obvious, we first explain some reformulations of Theorem 1 and point out a few applications showing that the formula (3) gives additional information about local symmetry of measures. The proof of the theorem is deferred to Sections 2 to 4 .

Measure distributions fulfilling (3) are well known in the theory of random measures. By a theorem of Mecke (see [Me]) the formula (3) characterizes $P$ as a Palm measure. Palm measures appear in many areas of probability, for example in queuing theory or in the theory of point processes, where they play the rôle of conditional distributions of stationary point processes given a point at the origin (see [K, Chap. 10]).

A measure distribution $P$ on $\mathcal{M}\left(\mathbf{R}^{d}\right)$ is a Palm measure if there is a stationary $\sigma$-finite measure $Q$ on $\mathcal{M}\left(\mathbf{R}^{d}\right)$ with finite intensity and

$$
\int_{M} \nu(B) d Q(\nu)=\int_{B} P \circ T^{u}(M) d u \text { for all } M \subseteq \mathcal{M}\left(\mathbf{R}^{d}\right), B \subseteq \mathbf{R}^{d} \text { Borel. }
$$

Mecke's characterization theorem states:

Lemma 9. A measure distribution $P$ on $\mathcal{M}\left(\mathbf{R}^{d}\right)$ is a Palm measure if and only if $P(\{\phi\})=0$, where $\phi$ is the zero-measure, and the Palm formula (3) holds.

Clearly $P(\{\phi\})=0$ for all $P \in \mathcal{P}^{\alpha}(\mu, x)$ if the lower $\alpha$-dimensional density of $\mu$ at $x$ is positive. Thus our main result implies that

Theorem 2. Let $\alpha \geq 0$ and $\mu \in \mathcal{M}\left(\mathbf{R}^{d}\right)$ be any measure. Then, at $\mu$-almost every point $x$ at which the lower $\alpha$-dimensional density of $\mu$ is positive, every tangent measure distribution $P \in \mathcal{P}^{\alpha}(\mu, x)$ is a Palm measure.

This statement establishes an interesting connection to the theory of self similar random measures developed by Ulrich Zähle in [ZU]. He suggested, heuristically speaking, that a random measure is statistically self similar if it is scaling invariant with respect to its "typical point" and we can interpret Palm measures as those measure distributions which have the origin as a "typical point". More precisely, a measure distribution $P$ on $\mathcal{M}\left(\mathbf{R}^{d}\right)$ is an $\alpha$-self similar random measure if $P$ is a Palm measure invariant under the rescaling group $\left(S_{\lambda}^{\alpha}\right)_{\lambda>0}$.

This concept has been subject of investigations by Patzschke, U. Zähle and M. Zähle, see for example [PaZU] and [PaZM]. In particular, they have studied the relation of the concept to statistically self similar measures in the constructive sense. Using the notion of $\alpha$-self similar random measures, we may reformulate Theorem 1 as

Theorem 3. Let $\alpha \geq 0$ and $\mu \in \mathcal{M}\left(\mathbf{R}^{d}\right)$ be any measure. Then, at $\mu$-almost all points $x$, every tangent measure distribution $P \in \mathcal{P}^{\alpha}(\mu, x)$ defines an $\alpha$-self similar random measure.

Here we have extended the notion of $\alpha$-self similar random measures to allow, in the natural way, for the possibility that $P(\{\phi\})>0$ and/or that the total mass of $P$ may be less than one. Of course, no such extension is needed if $\mu$ is supposed to have positive lower and finite upper $\alpha$-dimensional densities. 
Applications to average densities

Without further assumptions on the measure, the connection between average densities and tangent measure distributions is not straightforward, since it involves passing to a limit of an integral of the unbounded function $\nu \mapsto \nu U(0,1)$. For our purposes, the following information will suffice.

Proposition 5. Suppose that $\mu \in \mathcal{M}\left(\mathbf{R}^{d}\right), \alpha \geq 0, \delta_{n} \downarrow 0$ and that $P_{\delta_{n}}^{x}$ converge vaguely to a measure distribution $P$. If

$$
\liminf _{n \rightarrow \infty}\left(\left|\log \delta_{n}\right|\right)^{-1} \int_{\delta_{n}}^{1} \frac{\mu U(x, r)}{r^{\alpha}} \frac{d r}{r}<\infty
$$

then $P_{\delta_{n}}^{x}$ converge to $P$ weakly, hence $P$ is a probability measure and, for every $R>0$,

$$
\int \nu U(0, R) d P(\nu) \leq \liminf _{n \rightarrow \infty}\left(\left|\log \delta_{n}\right|\right)^{-1} \int_{\delta_{n}}^{1} \frac{\mu U(x, R r)}{r^{\alpha}} \frac{d r}{r} .
$$

If $\mu$ has finite upper $\alpha$-dimensional density at $x$, then there is a compact subset of $\mathcal{M}\left(\mathbf{R}^{d}\right)$ containing the supports of all measures $P_{\delta}^{x}, 0<\delta \leq 1$. In particular, $P$ is a probability measure and, for every $R>0$,

$$
\int \nu U(0, R) d P(\nu)=\lim _{n \rightarrow \infty}\left(\left|\log \delta_{n}\right|\right)^{-1} \int_{\delta_{n}}^{1} \frac{\mu U(x, R r)}{r^{\alpha}} \frac{d r}{r} .
$$

Proof. As the sequence $P_{\delta_{n}}^{x}$ converges weakly if a subsequence converges weakly, we may assume that the limit in (4) exists. For any $m=1,2, \ldots$ we infer from

$$
\begin{aligned}
\int \nu U(0, m) d P_{\delta_{n}}^{x}(\nu)= & \left(\left|\log \delta_{n}\right|\right)^{-1} \int_{\delta_{n}}^{1} \frac{\mu U(x, m r)}{r^{\alpha}} \frac{d r}{r} \\
= & m^{\alpha}\left(\left|\log \delta_{n}\right|\right)^{-1} \int_{m \delta_{n}}^{m} \frac{\mu U(x, r)}{r^{\alpha}} \frac{d r}{r} \\
\leq & m^{\alpha}\left(\left|\log \delta_{n}\right|\right)^{-1} \int_{\delta_{n}}^{1} \frac{\mu U(x, r)}{r^{\alpha}} \frac{d r}{r} \\
& +m^{\alpha}\left(\left|\log \delta_{n}\right|\right)^{-1} \int_{1}^{m} \frac{\mu U(x, r)}{r^{\alpha}} \frac{d r}{r}
\end{aligned}
$$

that the sequence $\int \nu U(0, m) d P_{\delta_{n}}^{x}(\nu), n=1,2, \ldots$ is bounded by, say, $C_{m}$. Thus, for any $\varepsilon>0$, the measures $P_{\delta_{n}}^{x}$ are concentrated, up to measure $\varepsilon$, on the compact set

$$
\bigcap_{m=1}^{\infty}\left\{\nu \in \mathcal{M}\left(\mathbf{R}^{d}\right): \nu U(0, m) \leq 2^{m} C_{m} / \varepsilon\right\}
$$

which shows that the vaguely convergent sequence $P_{\delta_{n}}^{x}$ converges weakly. The inequality (5) follows, since the function $\nu \mapsto \nu U(0, R)$ is lower semi-continuous. If $\mu$ has finite upper $\alpha$-dimensional density at $x$, then the set $\left\{\mu_{x, r} / r^{\alpha}: 0<r \leq\right.$ $1\}$ is relatively compact in $\mathcal{M}\left(\mathbf{R}^{d}\right)$; its closure has the required property. Thus 
the function $\nu \mapsto \nu \overline{U(0, R)}$ is upper semi-continuous and bounded on a compact set containing supports of all $P_{\delta_{n}}^{x}$. Hence

$$
\begin{aligned}
\int \nu \overline{U(0, R)} d P(\nu) & \geq \limsup _{n \rightarrow \infty}\left(\left|\log \delta_{n}\right|\right)^{-1} \int_{\delta_{n}}^{1} \frac{\mu \overline{U(x, R r)}}{r^{\alpha}} \frac{d r}{r} \\
& \geq \liminf _{n \rightarrow \infty}\left(\left|\log \delta_{n}\right|\right)^{-1} \int_{\delta_{n}}^{1} \frac{\mu U(x, R r)}{r^{\alpha}} \frac{d r}{r} \\
& \geq \int \nu U(0, R) d P(\nu)=\int \nu \overline{U(0, R)} d P(\nu),
\end{aligned}
$$

using Proposition 4 in the last step. This proves Proposition 5.

We may use the formula (3) of Theorem 1 with $G(\nu, u)=h(u)$ to infer that, at $\mu$-almost all points $x$, every tangent measure distribution $P \in \mathcal{P}^{\alpha}(\mu, x)$ fulfills

$$
\iint h(u) d \nu(u) d P(\nu)=\iint h(-u) d \nu(u) d P(\nu)
$$

for every Borel function $h: \mathbf{R}^{d} \longrightarrow[0, \infty)$. Choosing for $h$ characteristic functions only, we get

$$
\int \nu(U) d P(\nu)=\int \nu(-U) d P(\nu)
$$

for every Borel subset $U$ of $\mathbf{R}^{d}$. A number of unexpected statements concerning average densities, which have no analogy for ordinary densities, follow from this equality.

Proposition 6. If $\mu$ has finite upper $\alpha$-dimensional density almost everywhere, then at $\mu$-almost all points $x$ the following holds: Whenever $U \subseteq V \subseteq \mathbf{R}^{d}$ with $U$ closed and $V$ open and $\delta_{n} \downarrow 0$, we have

$$
\liminf _{n \rightarrow \infty}\left(\left|\log \delta_{n}\right|\right)^{-1} \int_{\delta_{n}}^{1} \frac{\mu(x+r U)}{r^{\alpha}} \frac{d r}{r} \leq \liminf _{n \rightarrow \infty}\left(\left|\log \delta_{n}\right|\right)^{-1} \int_{\delta_{n}}^{1} \frac{\mu(x-r V)}{r^{\alpha}} \frac{d r}{r} .
$$

Proof. We may assume that $P_{\delta_{n}}^{x}$ converges to a measure distribution $P$. Employing the compactness result of Proposition 5, we infer that

$$
\begin{aligned}
\liminf _{n \rightarrow \infty}\left(\left|\log \delta_{n}\right|\right)^{-1} \int_{\delta_{n}}^{1} \frac{\mu(x+r U)}{r^{\alpha}} \frac{d r}{r} \\
\quad \leq \int \nu(U) d P(\nu)=\int \nu(-U) d P(\nu) \\
\quad \leq \int \nu(-V) d P(\nu) \\
\quad \leq \liminf _{n \rightarrow \infty}\left(\left|\log \delta_{n}\right|\right)^{-1} \int_{\delta_{n}}^{1} \frac{\mu(x-r V)}{r^{\alpha}} \frac{d r}{r}
\end{aligned}
$$


Whenever $C$ is a Borel cone in $\mathbf{R}^{d}$, one defines the lower average $\alpha$-dimensional density of a measure $\mu$ at a point $x$ with respect to $C$ as

$$
\liminf _{\delta \downarrow 0}(|\log \delta|)^{-1} \int_{\delta}^{1} \frac{\mu((x+C) \cap U(x, r))}{r^{\alpha}} \frac{d r}{r} .
$$

If $C=\mathbf{R}^{d}$, this is the lower average density of the measure $\mu$ at a point $x$, if $d=1$ and $C=(0, \infty)$ we speak about right lower average density of the measure $\mu$ at a point $x$, etc.

Proposition 7. Suppose $\alpha>0, \mu \in \mathcal{M}\left(\mathbf{R}^{d}\right)$ has finite upper $\alpha$-dimensional density almost everywhere and $C$ is a closed cone which (except for the origin) is contained in the open cone $K$. Then, at $\mu$-almost all points $x$, the lower average $\alpha$-dimensional density of the measure $\mu$ with respect to $C$ does not exceed its lower average $\alpha$-dimensional density with respect to the cone $-K$.

Proof. For every tangent measure distribution $P$ the scaling invariance property implies that $\int \nu(\overline{U(0,1)} \cap C) d P(\nu) \leq \int \nu(U(0,1) \cap K) d P(\nu)$ and we can employ the same argument as in Proposition 6.

As an immediate corollary of these propositions we obtain the following result, which was obtained only under the additional assumption of positive lower $\alpha$ dimensional density in [Mö2] and, in a weaker form, in [MöPr].

Theorem 4. Let $\alpha>0$ and $\mu \in \mathcal{M}(\mathbf{R})$ be a measure on the line with finite upper $\alpha$-dimensional densities $\mu$-almost everywhere. Then, at $\mu$-almost all points $x$, the right and left lower average $\alpha$-dimensional densities coincide and each of them is half of the lower average $\alpha$-dimensional density of $\mu$ at $x$.

Of course, analogous results can be found for upper average densities.

As our final application we show in Theorem 5 how the results of [FaSp] and [Ma2] follow naturally by using the higher regularity of tangent measure distributions. Our approach removes their unnatural assumption of finiteness of upper densities for results about lower densities.

Lemma 10. Suppose that a probability measure $P$ on $\mathcal{M}\left(\mathbf{R}^{d}\right)$ defines an $\alpha$-self similar random measure and that, with some $c>0, P$-almost every $\nu$ satisfies $\nu U(0,1)=c$. Then $\alpha$ is an integer.

Proof. From the invariance of $P$ under the rescaling group $\left(S_{\lambda}^{\alpha}\right)_{\lambda>0}$ we infer that, for every $r>0$ and $P$-almost every $\nu$, we have $\nu U(0, r)=c r^{\alpha}$. Thus, using the Palm formula in the last step,

$$
\begin{aligned}
1 & =P\left(\left\{\mu: \mu U(0, r)=c r^{\alpha}\right\}\right)=\frac{1}{c s^{\alpha}} \iint_{U(0, s)} 1_{\left\{\mu: \mu U(0, r)=c r^{\alpha}\right\}}(\nu) d \nu(u) d P(\nu) \\
& =\frac{1}{c s^{\alpha}} \iint_{U(0, s)} 1_{\left\{\mu: \mu U(u, r)=c r^{\alpha}\right\}}(\nu) d \nu(u) d P(\nu) .
\end{aligned}
$$

As $r$ and $s$ are arbitrary, for $P$-almost every $\nu$ we infer that $\nu U(u, r)=c r^{\alpha}$ for $\nu$-almost every $u$ and every rational $r>0$. By continuity, $P$-almost every $\nu$ 
satisfies that $\nu U(u, r)=c r^{\alpha}$ for every $u$ in the support of $\nu$ and every $r>0$. Now it suffices to use Marstrand's Theorem ([Ma1], see also [Mat, 14.10]) to infer that $\alpha$ is an integer.

Theorem 5. Suppose that $\mu \in \mathcal{M}\left(\mathbf{R}^{d}\right)$ and that $\alpha>0$ is not an integer. Then $\mu$-almost all points $x$ satisfy:

(a) The lower average $\alpha$-dimensional density and the lower $\alpha$-dimensional density of $\mu$ at $x$ are either both zero, or both infinite, or the former is strictly bigger than the latter.

(b) The upper average $\alpha$-dimensional density and the upper $\alpha$-dimensional density of $\mu$ at $x$ are either both zero, or both infinite, or the former is strictly smaller than the latter.

Proof. If $d(x)$ and $D(x)$ denote the (ordinary) lower and upper $\alpha$-dimensional densities of $\mu$, respectively, then, for $\mu$-almost every $x$, each $\nu \in \operatorname{Tan}_{\alpha}(\mu, x)$ satisfies $d(x) \leq \nu U(0,1) \leq D(x)$. To use Lemma 10, it suffices to observe that if the average and ordinary lower, respectively upper, densities of $\mu$ at $x$ coincide and are finite, then there is a probability measure $P \in \mathcal{P}^{\alpha}(\mu, x)$ such that $P$ almost every $\nu$ satisfies that $\nu U(0,1)=d(x)$, respectively $\nu U(0,1)=D(x)$. But if $d(x)$, respectively $D(x)$, is finite, then we can find $\delta_{n} \downarrow 0$ such that

$$
\lim _{n \rightarrow \infty}\left(\left|\log \delta_{n}\right|\right)^{-1} \int_{\delta_{n}}^{1} \frac{\mu U(x, r)}{r^{\alpha}} \frac{d r}{r}=d(x) \text {, respectively } D(x) .
$$

We can choose $P$ as the limit of a subsequence of $P_{\delta_{n}}^{x}$. Then, by Proposition 1(a), $d(x) \leq \nu U(0,1) \leq D(x)$ for $P$-almost every $\nu$, and we may use the inequality (5) of Proposition 5 in case of lower densities and the equality (6) of the same proposition in case of upper densities to infer that $P$-almost every $\nu$ satisfies $\nu U(0,1)=d(x)$, respectively $\nu U(0,1)=D(x)$.

\section{The main lemma}

Although our results do not depend on the choice of the norm of $\mathbf{R}^{d}$, it will be convenient to equip it with the maximum norm defined, for $u=\left(u_{1}, \ldots, u_{d}\right)$, by

$$
\|u\|=\max \left\{\left|u_{i}\right|: i=1, \ldots, d\right\}
$$

and denote by $B(u, r)$ the closed balls with respect to this norm, i.e., $B(u, r)=$ $\left\{x \in \mathbf{R}^{d}:\|u-x\| \leq r\right\}$.

Lemma 11. Whenever a measure $\mu \in \mathcal{M}\left(\mathbf{R}^{d}\right)$, a Borel measurable function $H: \mathbf{R}^{d} \times \mathbf{R}^{d} \times(0, \infty) \longrightarrow[0,1]$ and $0<\alpha<d$ satisfy, for some constants $R, A_{1}, A_{2} \in(0, \infty)$, the conditions

(i) $H(x, y ; r)=0$ whenever $\|x-y\| \geq R r$,

(ii) $H(x, y ; r)=0$ whenever $\mu B(x, 5 R r) \geq A_{1} r^{\alpha}$, and

(iii) for all $x, \tilde{x}, y, \tilde{y} \in \mathbf{R}^{d}$ and $0<r<\infty$,

$$
|H(x, y ; r)-H(\tilde{x}, \tilde{y} ; r)| \leq A_{2} \frac{\|x-\tilde{x}\|+\|y-\tilde{y}\|}{r},
$$


then, for $\mu$-almost every $x \in \mathbf{R}^{d}$,

$$
\lim _{\delta \downarrow 0} \frac{1}{|\log \delta|} \int_{\delta}^{1} \int \frac{H(x, y ; r)-H(y, x ; r)}{r^{\alpha}} d \mu(y) \frac{d r}{r}=0 .
$$

The rest of this section is devoted to the proof of this lemma. Our plan is first to find a dyadic model of the statement of the lemma, then show how this approximation may be used to prove its statement, and only then to embark upon the series of technical estimates which will eventually result in the proof of the dyadic version of our main lemma.

\section{The dyadic model}

We approximate the situation of Lemma 11 by discretizing it in a dyadic grid. For $k=0,1, \ldots$, we define the $k$-th grid as the family of dyadic cubes

$$
\mathcal{Q}_{k}=\left\{\prod_{i=1}^{d}\left[\frac{k_{i}}{2^{k}}, \frac{k_{i}+1}{2^{k}}\right):\left(k_{1}, \ldots, k_{d}\right) \in \mathbf{Z}^{d}\right\} .
$$

Any $x \in \mathbf{R}^{d}$ is contained in a unique cube from $\mathcal{Q}_{k}$; this cube will be denoted by $Q_{k}(x)$. The dyadic approximation to our main lemma is given in the following statement.

Lemma 12. Given any $\sigma>0,0<\alpha<d$, and constants $C_{1}, C_{2} \in(0, \infty)$, we can find a constant $\mathcal{C}_{0}=\mathcal{C}_{0}\left(\sigma, \alpha, d, C_{1}, C_{2}\right) \in(0, \infty)$ having the following property: Whenever a finite measure $\mu \in \mathcal{M}\left(\mathbf{R}^{d}\right)$ and Borel measurable functions $H_{k}: \mathbf{R}^{d} \times \mathbf{R}^{d} \longrightarrow \mathbf{R}$ satisfy the conditions

(i) $H_{k}(x, y)=0$ whenever $y \notin Q_{k}(x)$,

(ii) $H_{k}(x, y)=0$ whenever $\mu\left(Q_{k}(x)\right)>C_{1} 2^{-k \alpha}$,

(iii) $\left|H_{k}(x, u)-H_{k}(y, u)\right| \leq C_{2} 2^{k \alpha+k-i}$ if $i \geq k, x, y \in I \in \mathcal{Q}_{i}$ and $u \in Q_{k}(x)$,

(iv) $H_{k}(y, x)=-H_{k}(x, y)$ for all $k=0,1, \ldots$ and all $x, y \in \mathbf{R}^{d}$,

then for any $n=1,2, \ldots$ and any non-negative function $g \in L_{2}(\mu)$,

$$
\int g(x)\left(\int \sum_{k=0}^{n-1} H_{k}(x, u) d \mu(u)-\sigma n\right) d \mu(x) \leq \mathcal{C}_{0} n^{3 / 4} \mu\left(\mathbf{R}^{d}\right)^{1 / 2}\|g\|_{L_{2}(\mu)} .
$$

Although we shall not need it, it is interesting to note that our proof allows one to describe the dependence of $\mathcal{C}_{0}$ on all parameters explicitly; this shows, in particular, that $\mathcal{C}_{0}$ may be chosen independently of $\alpha$ as long as $\alpha$ is kept away from $d$. 
Proof of Lemma 11 using Lemma 12

We need only prove Lemma 11 under the additional assumption that $\mu$ is finite, as the general case immediately follows by using the special one for the restrictions of $\mu$ to $B(0, k), k=1,2, \ldots$.

Lemma 13. Suppose that $0<\alpha<d, \mu \in \mathcal{M}\left(\mathbf{R}^{d}\right)$ is a finite measure and $G: \mathbf{R}^{d} \times \mathbf{R}^{d} \times(0, \infty) \longrightarrow \mathbf{R}$ is a Borel measurable function, and $\hat{A}_{1}, \hat{A}_{2} \in(0, \infty)$ are constants such that

(i) $|G(x, y ; r)| \leq r^{-\alpha}$ for all $x, y \in \mathbf{R}^{d}$ and $r>0$,

(ii) $G(x, y ; r)=0$ whenever $\|x-y\| \geq r$,

(iii) $G(x, y ; r)=0$ whenever $\mu B(x, 4 r) \geq \hat{A}_{1} r^{\alpha}$,

(iv) for all $x, y, u \in \mathbf{R}^{d}$ and $0<r<\infty$,

$$
|G(x, u ; r)-G(y, u ; r)| \leq \hat{A}_{2} r^{-\alpha-1}\|x-y\|,
$$

(v) $G(y, x ; r)=-G(x, y ; r)$ for all $x, y \in \mathbf{R}^{d}$ and $r>0$.

Then for every $\sigma>0$ there is $\mathcal{C}_{1}<\infty$ such that for any $n=1,2, \ldots$ and any non-negative $g \in L_{2}(\mu)$,

$$
\int g(x)\left[\int_{2^{-n-1}}^{1 / 2} \int G(x, y ; r) d \mu(y) \frac{d r}{r}-\sigma n\right] d \mu(x) \leq \mathcal{C}_{1} n^{3 / 4}\|g\|_{L_{2}(\mu)} .
$$

Consequently, for $\mu$-almost all $x \in \mathbf{R}^{d}$, the equality

$$
\lim _{\delta \downarrow 0} \frac{1}{|\log \delta|} \int_{\delta R}^{R} \int G(x, y ; r) d \mu(y) \frac{d r}{r}=0
$$

holds for every $R>0$.

Proof. The function

$$
\omega(z)=\prod_{i=1}^{d}\left(1-\min \left\{\left|z_{i}\right|, 1 / 2\right\}\right)^{-1}
$$

verifies $1 \leq \omega(z) \leq 2^{d}$ and is Lipschitz on $\mathbf{R}^{d}$; let $C_{0}$ be its Lipschitz constant. We define $C_{1}=\hat{A}_{1}$ and $C_{2}=4^{\alpha} C_{0}+2^{d+2 \alpha+2} \hat{A}_{2}$, and we prove that, for any $a \in \mathbf{R}^{d}$ and any $1 / 4 \leq s \leq 1 / 2$, the functions

$$
H_{k}(x, y ; a, s)=\sum_{Q \in \mathcal{Q}_{k}} 1_{Q}(x) 1_{Q}(y) G\left(a+x, a+y ; 2^{-k} s\right) \omega\left(2^{k}(y-x)\right)
$$

satisfy the assumptions (i)-(iv) of Lemma 12 for the measure $T^{a} \mu$. Clearly, $12(\mathrm{i})$ follows directly from the definition of $H_{k}(x, y ; a, s)$ and 12 (iv) follows directly from $13(\mathrm{v})$. If $T^{a} \mu\left(Q_{k}(x)\right)>C_{1} 2^{-k \alpha}$, then

$$
\begin{aligned}
\mu\left(B\left(a+x, 2^{-k+2} s\right)\right) & \geq \mu\left(B\left(a+x, 2^{-k}\right)\right) \geq \mu\left(a+Q_{k}(x)\right)=T^{a} \mu\left(Q_{k}(x)\right) \\
& >C_{1} 2^{-k \alpha} \geq \hat{A}_{1}\left(2^{-k} s\right)^{\alpha},
\end{aligned}
$$


which, by $13\left(\right.$ iii), shows that $G\left(a+x, a+y ; 2^{-k} s\right)=0$ and hence the requirement of 12 (ii) that $H_{k}(x, y ; a, s)=0$. Finally, the condition 12 (iii) is also easy, since $i \geq k, x, y \in I \in \mathcal{Q}_{i}$ and $u \in Q_{k}(x)$ imply, because of 13(ii) and 13(iv), that

$$
\begin{aligned}
& \left|H_{k}(x, u ; a, s)-H_{k}(y, u ; a, s)\right| \\
& \quad=\left|G\left(x+a, u+a ; 2^{-k} s\right) \omega\left(2^{k}(u-x)\right)-G\left(y+a, u+a ; 2^{-k} s\right) \omega\left(2^{k}(u-y)\right)\right| \\
& \leq\left|\omega\left(2^{k}(u-x)\right)-\omega\left(2^{k}(u-y)\right)\right|\left|G\left(x+a, u+a ; 2^{-k} s\right)\right| \\
& \quad+\left|\omega\left(2^{k}(u-y)\right)\right|\left|G\left(x+a, u+a ; 2^{-k} s\right)-G\left(y+a, u+a ; 2^{-k} s\right)\right| \\
& \quad \leq \quad\left(2^{-k} s\right)^{-\alpha} C_{0} 2^{k}|| y-x\left\|+2^{d} \hat{A}_{2}\left(2^{-k} s\right)^{-\alpha-1}\right\| x-y \| \\
& \leq C_{2} 2^{k \alpha+k-i} .
\end{aligned}
$$

Let $\mathcal{C}=\mathcal{C}_{0}\left(\sigma / \log 2, \alpha, d, C_{1}, C_{2}\right) \mu\left(\mathbf{R}^{d}\right)^{1 / 2}$, where $\mathcal{C}_{0}$ is the constant introduced in Lemma 12 and let $g \in L_{2}(\mu)$ be non-negative. The statement of Lemma 12 then implies that, for every $a \in \mathbf{R}^{d}$ and every $1 / 4 \leq s \leq 1 / 2$,

$$
\begin{gathered}
\int g(x+a)\left(\int \sum_{k=0}^{n-1} H_{k}(x, u ; a, s) d T^{a} \mu(u)-\sigma n / \log 2\right) d T^{a} \mu(x) \\
\leq \mathcal{C} n^{3 / 4}\|g\|_{L_{2}(\mu)} .
\end{gathered}
$$

Hence, denoting

$$
\begin{aligned}
G_{k}(x, y ; a, s) & =H_{k}(x-a, y-a ; a, s) \\
& =\sum_{Q \in \mathcal{Q}_{k}} 1_{a+Q}(x) 1_{a+Q}(y) G\left(x, y ; 2^{-k} s\right) \omega\left(2^{k}(y-x)\right),
\end{aligned}
$$

we have that

$$
\int g(x)\left(\int \sum_{k=0}^{n-1} G_{k}(x, u ; a, s) d \mu(u)-\sigma n / \log 2\right) d \mu(x) \leq \mathcal{C} n^{3 / 4}\|g\|_{L_{2}(\mu)} .
$$

If $\|y-x\| \leq 2^{-k} s$, we use that $s \leq 1 / 2$ to infer that $\omega\left(2^{k}(y-x)\right)=\prod_{i=1}^{d}(1-$ $\left.2^{k}\left|y_{i}-x_{i}\right|\right)^{-1}$. Thus

$$
\begin{aligned}
& \int_{[0,1]^{d}} \sum_{Q \in \mathcal{Q}_{k}} 1_{a+Q}(x) 1_{a+Q}(y) d \mathcal{L}^{d}(a) \\
& \quad=\mathcal{L}^{d}\left\{a \in[0,1]^{d}: \sum_{Q \in \mathcal{Q}_{k}} 1_{a+Q}(x) 1_{a+Q}(y)=1\right\} \\
& \quad=1 / \omega\left(2^{k}(y-x)\right)
\end{aligned}
$$

and we conclude that

$$
G\left(x, y ; 2^{-k} s\right)=\int_{[0,1]^{d}} G_{k}(x, y ; a, s) d \mathcal{L}^{d}(a) .
$$

If $\|y-x\|>2^{-k} s$, then both sides of (11) are zero according to (ii); hence (11) may be used for all $x, y$. Thus, integrating $(10)$ over $[0,1]^{d}$ with respect to the 
Lebesgue measure and over $1 / 4 \leq s \leq 1 / 2$ with respect to the measure $\frac{d s}{s}$ and using that

$$
\int_{1 / 4}^{1 / 2} G\left(x, u ; 2^{-k} s\right) \frac{d s}{s}=\int_{2^{-k-2}}^{2^{-k-1}} G(x, u ; r) \frac{d r}{r}
$$

we get

$$
\int g(x)\left[\int_{2^{-n-1}}^{1 / 2} \int G(x, y ; r) d \mu(y) \frac{d r}{r}-\sigma n\right] d \mu(x) \leq \mathcal{C} n^{3 / 4}\|g\|_{L_{2}(\mu)} \log 2,
$$

which shows that (8) holds with $\mathcal{C}_{1}=\mathcal{C} \log 2$.

To prove (9), we denote

$$
\bar{G}(x ; r)=\int G(x, y ; r) d \mu(y)
$$

and note that, by $13(\mathrm{ii}), 13(\mathrm{v})$ and $13(\mathrm{iv})$,

$$
\begin{aligned}
|G(x, y ; r)| & =1_{B(x, r)}(y)|G(x, y ; r)-G(y, y ; r)| \leq \hat{A}_{2} r^{-\alpha-1}\|x-y\| 1_{B(x, r)}(y) \\
& \leq \hat{A}_{2} r^{-\alpha} 1_{B(x, r)}(y) .
\end{aligned}
$$

Since, by 13 (iii), the left side is zero unless $\mu(B(x, r)) \leq \hat{A}_{1} r^{\alpha}$, we obtain by integrating with respect to $d \mu(y)$ that for all $x$ and $r$,

$$
|\bar{G}(x ; r)| \leq \hat{A}_{2} r^{-\alpha} \mu(B(x, r)) \leq \hat{A}_{1} \hat{A}_{2} .
$$

Next we use (8) for the characteristic function $g$ of the set

$$
E_{n}=\left\{x: \int_{2^{-n-1}}^{1 / 2} \bar{G}(x ; r) \frac{d r}{r}>2 \sigma n\right\}
$$

to get that

$$
\sigma n \mu\left(E_{n}\right) \leq \mathcal{C}_{1} n^{3 / 4} \mu\left(E_{n}\right)^{1 / 2},
$$

hence $\mu\left(E_{n}\right) \leq \mathcal{C}_{1}^{2} n^{-1 / 2} / \sigma^{2}$, which shows that $\sum_{n=1}^{\infty} \mu\left(E_{n^{3}}\right)$ converges. By the Borel-Cantelli Lemma, for $\mu$-almost every $x \in \mathbf{R}^{d}$ there is a positive integer $m$ such that $x \notin \bigcup_{n=m}^{\infty} E_{n^{3}}$. Consider any such $x$ and the corresponding $m$; we may clearly also assume that $m \geq 7 \hat{A}_{1} \hat{A}_{2} / \sigma$. Whenever $0<\delta<2^{-m^{3}}$ we find $n \geq m$ such that $2^{-(n+1)^{3}} \leq \delta<2^{-n^{3}}$ and we note that $x \notin E_{n^{3}}$ implies that

$$
\frac{1}{|\log \delta|} \int_{2^{-n^{3}-1}}^{1 / 2} \bar{G}(x ; r) \frac{d r}{r}=\frac{n^{3}}{|\log \delta|} \frac{1}{n^{3}} \int_{2^{-n^{3}-1}}^{1 / 2} \bar{G}(x ; r) \frac{d r}{r} \leq 2 \sigma / \log 2 .
$$

Since (12) gives

$$
\frac{1}{|\log \delta|} \int_{1 / 2}^{R} \bar{G}(x ; r) \frac{d r}{r} \leq \frac{\hat{A}_{1} \hat{A}_{2}(|\log R|+\log 2)}{|\log \delta|}
$$

and 


$$
\begin{aligned}
\frac{1}{|\log \delta|} \int_{\delta R}^{2^{n^{3}-1}} \bar{G}(x ; r) \frac{d r}{r} & \leq \frac{\hat{A}_{1} \hat{A}_{2}\left(|\log R|+\left((n+1)^{3}-n^{3}\right) \log 2\right)}{|\log \delta|} \\
& \leq \frac{\hat{A}_{1} \hat{A}_{2}|\log R|}{|\log \delta|}+\frac{7 \hat{A}_{1} \hat{A}_{2}}{n},
\end{aligned}
$$

we infer that

$$
\limsup _{\delta \downarrow 0} \frac{1}{|\log \delta|} \int_{\delta R}^{R} \int G(x, y ; r) d \mu(y) \frac{d r}{r} \leq 2 \sigma / \log 2
$$

for every $R>0$. Since $\sigma>0$ may be arbitrary, it follows that for $\mu$-almost every $x$ the inequality

$$
\limsup _{\delta \downarrow 0} \frac{1}{|\log \delta|} \int_{\delta R}^{R} \int G(x, y ; r) d \mu(y) \frac{d r}{r} \leq 0
$$

holds for every $R>0$. The statement (9) follows by using this also with $G$ replaced by $-G$.

Proof of Lemma 11. If the assumptions of Lemma 11 hold, we let $\hat{A}_{1}=A_{1} R^{-\alpha}$, $\hat{A}_{2}=2 A_{2} R$, and

$$
G(x, y ; r)=\frac{H(x, y ; r / R)-H(y, x ; r / R)}{r^{\alpha}},
$$

and note that the assumptions (i), (ii), (iv) and (v) of Lemma 13 are clearly satisfied. To establish 13(iii), we observe that it follows directly from 11(i) if $\|x-y\| \geq r$. If $\|x-y\|<r$ then $B(x, 5 r) \cap B(y, 5 r) \supseteq B(x, 4 r)$, hence $\mu B(x, 4 r) \geq$ $\hat{A}_{1} r^{\alpha}$ implies that $\mu B(x, 5 r) \geq A_{1}(r / R)^{\alpha}$ and $\mu B(y, 5 r) \geq A_{1}(r / R)^{\alpha}$, hence $H(x, y ; r / R)=H(y, x ; r / R)=0$ according to 11(ii) .

Hence, using (9) of Lemma 13, we have

$$
\begin{aligned}
& \lim _{\delta \downarrow 0} \frac{1}{|\log \delta|} \int_{\delta}^{1} \int \frac{H(x, y ; r)-H(y, x ; r)}{r^{\alpha}} d \mu(y) \frac{d r}{r} \\
& \quad=\quad R^{\alpha} \lim _{\delta \downarrow 0} \frac{1}{|\log \delta|} \int_{\delta R}^{R} \int G(x, y ; r) d \mu(y) \frac{d r}{r}=0 .
\end{aligned}
$$

\section{Proof of Lemma 12}

For the remainder of this section we shall assume that $0<\alpha<d, \sigma, C_{1}, C_{2}>0$, the measure $\mu$, the functions $H_{k}$ and an integer $n$ are fixed. Define the cut-off function

$$
\omega_{k}(x)= \begin{cases}1 & \text { if } C_{2}^{-1} \sigma 2^{-k \alpha} \leq \mu\left(Q_{k}(x)\right) \leq C_{1} 2^{-k \alpha}, \\ 0 & \text { otherwise }\end{cases}
$$

and let, for $k \in\{0,1, \ldots, n-1\}, x \in \mathbf{R}^{d}$ and Borel sets $E \subseteq \mathbf{R}^{d}$, 


$$
H_{k}(x)=\omega_{k}(x) \int_{Q_{k}(x)} H_{k}(x, u) d \mu(u)
$$

and

$$
\varphi_{x}(E)=\left(C_{1} C_{2}\right)^{-1} \sum_{k=0}^{n-1} \frac{\mathcal{L}^{d}\left(E \cap\left(Q_{k}(x) \backslash Q_{k+1}(x)\right)\right)}{\mathcal{L}^{d}\left(Q_{k}(x) \backslash Q_{k+1}(x)\right)} H_{k}(x) .
$$

These notions are justified by the following observation.

Lemma 14. For every positive function $g \in L_{2}(\mu)$,

$$
\begin{aligned}
& \int g(x)\left(\int \sum_{k=0}^{n-1} H_{k}(x, u) d \mu(u)-\sigma n\right) d \mu(x) \\
& \leq C_{1} C_{2} \int g(x) \varphi_{x}\left(\mathbf{R}^{d}\right) d \mu(x) \\
& \leq C_{1} C_{2}\|g\|_{L_{2}(\mu)}\left(\int\left(\varphi_{x}\left(\mathbf{R}^{d}\right)\right)^{2} d \mu(x)\right)^{1 / 2} .
\end{aligned}
$$

Proof. By (i) and by the case $i=k$ of (iii), $H_{k}(x, u) \leq C_{2} 2^{k \alpha} 1_{Q_{k}(x)}(u)$. Thus, if $\omega_{k}(x)=0$, then either $\mu\left(Q_{k}(x)\right)<C_{2}^{-1} \sigma 2^{-k \alpha}$ and $\int H_{k}(x, u) d \mu(u) \leq$ $C_{2} 2^{k \alpha} \mu\left(Q_{k}(x)\right)<\sigma$, or $\mu\left(Q_{k}(x)\right)>C_{1} 2^{-k \alpha}$ in which case we infer from (ii) that $\int H_{k}(x, u) d \mu(u)=0<\sigma$, and hence

$$
\int \sum_{k=0}^{n-1} H_{k}(x, u) d \mu(u)-\sigma n \leq \int \sum_{k=0}^{n-1} \omega_{k}(x) H_{k}(x, u) d \mu(u)=C_{1} C_{2} \varphi_{x}\left(\mathbf{R}^{d}\right) .
$$

The statement follows by multiplying this inequality by $g(x)$, integrating with respect to $d \mu(x)$, and using Hölder's inequality.

We therefore devote the rest of this section to the estimate of $\int\left(\varphi_{x}\left(\mathbf{R}^{d}\right)\right)^{2} d \mu(x)$. Since working with the signed measures $\varphi_{x}$ may sometimes be slightly awkward, we majorize them by Radon measures $\psi_{x}\left(x \in \mathbf{R}^{d}\right)$ defined by

$$
\psi_{x}(E)=\sum_{k=0}^{n-1} \frac{\mathcal{L}^{d}\left(E \cap\left(Q_{k}(x) \backslash Q_{k+1}(x)\right)\right)}{\mathcal{L}^{d}\left(Q_{k}(x) \backslash Q_{k+1}(x)\right)} \omega_{k}(x) .
$$

To see the majorization, we first note that $\omega_{k}(x) \neq 0$ implies that $\omega_{k}(x)=1$ and $\mu\left(Q_{k}(x)\right) \leq C_{1} 2^{-k \alpha}$, which proves that

Lemma 15. For every $x \in \mathbf{R}^{d}$ and every $k \in\{0,1, \ldots, n-1\}$,

$$
\omega_{k}(x) 2^{k \alpha} \mu\left(Q_{k}(x)\right) \leq C_{1} .
$$

Consequently, $\left|H_{k}(x)\right| \leq \omega_{k}(x) \int_{Q_{k}(x)}\left|H_{k}(x, u)\right| d \mu(u) \leq \omega_{k}(x) 2^{k \alpha} C_{2} \mu\left(Q_{k}(x)\right) \leq$ $\omega_{k}(x) C_{1} C_{2}$, and we infer that

Lemma 16. For every $x \in \mathbf{R}^{d}$ and every Borel set $E \subseteq \mathbf{R}^{d}$,

$$
\left|\varphi_{x}(E)\right| \leq \psi_{x}(E)
$$


In addition to the above auxiliary functions and measures, we need to decompose the family of dyadic cubes into several subfamilies. We denote by

$$
\mathcal{I}=\mathcal{Q}_{0} \cup \bigcup_{k=1}^{n-1}\left\{Q \in \mathcal{Q}_{k}: \mu(Q) \geq C_{2}^{-1} \sigma 2^{-k \alpha}\right\}
$$

the family of those dyadic cubes which have a non-negligible $\mu$ measure (or belong to the starting grid).

Noting that $Q_{0}(x) \in \mathcal{I}$ for every $x \in \mathbf{R}^{d}$, we define

$$
k(x)=\max \left\{k=0,1, \ldots, n-1: Q_{k}(x) \in \mathcal{I}\right\}
$$

and, for $I \in \mathcal{Q}_{m}$,

$$
G(I)=\{x \in I: k(x)=m\} .
$$

It will also be convenient to extend the notation $Q_{k}(x)$ in the following way: If $I \in \mathcal{Q}_{m}$ and $0 \leq k \leq m$, then $Q_{k}(I)$ will denote the unique cube from $\mathcal{Q}_{k}$ containing $I$.

Finally, we choose the smallest integer $q$ such that $q^{2} \geq n$, define, for $i \in$ $\{0,1, \ldots, q-1\}$,

$$
\mathcal{I}_{i}=\mathcal{I} \cap \bigcup_{k=i q}^{(i+1) q-1} \mathcal{Q}_{k}
$$

and denote by $\mathcal{K}_{i}$ the set of maximal cubes from the set $\mathcal{I} \cap \bigcup_{k=i q}^{n-1} \mathcal{Q}_{k}=\bigcup_{j=i}^{q-1} \mathcal{I}_{j}$, i.e.,

$$
\mathcal{K}_{i}=\left\{K \in \mathcal{I} \cap \bigcup_{k=i q}^{n-1} \mathcal{Q}_{k}: K \subseteq I \in \bigcup_{k=i q}^{n-1} \mathcal{Q}_{k}, K \neq I \text { implies } I \notin \mathcal{I}\right\} .
$$

Lemma 17. If $K \in \mathcal{I}$, the sets $G(I)(I \in \mathcal{I}, I \subseteq K)$ form a (Borel measurable, finite) partition of $K$. Consequently, the union of the family $\mathcal{K}_{i}$ is partitioned into the cubes from $\mathcal{K}_{i}$ as well as into the sets $G(I)\left(I \in \bigcup_{k=i}^{q-1} \mathcal{I}_{k}\right)$. In particular, the sets $G(I)(I \in \mathcal{I})$ form a (Borel measurable, locally finite) partition of $\mathbf{R}^{d}$.

Proof. Suppose that $K \in \mathcal{I} \cap \mathcal{Q}_{m}$. If $x \in K$, then $k(x) \geq m$, so $I=Q_{k(x)}(x) \subseteq$ $K, I \in \mathcal{I}$ and $x \in G(I)$. Hence $K \subseteq \bigcup_{I \in \mathcal{I}, I \subseteq K} G(I)$ which, since $G(I) \subseteq \bar{I}$, implies that $K=\bigcup_{I \in \mathcal{I}, I \subseteq K} G(I)$. Finally, $x \in G(I) \cap G(J)$ implies that $I=$ $Q_{k(x)}(x)=J$, so $G(I) \cap \bar{G}(J)=\emptyset$ for $I \neq J$. The bracketed statements about Borel measurability and finiteness are obvious since each $G(I)$ is a finite union of cubes from $\mathcal{Q}_{n-1}$ and the number of those $I \in \mathcal{Q}$ for which $I \subseteq K$ is finite.

Since the cubes from the family $\mathcal{K}_{i}$ are clearly disjoint, their union is partitioned by them as well as by the sets $G(I)$, where $I$ runs through cubes from $\mathcal{I}$ that are subsets of cubes from $\mathcal{K}_{i}$. But each such $I$ belongs to $\bigcup_{k=i}^{q-1} \mathcal{I}_{k}$ and, conversely, each $I \in \bigcup_{k=i}^{q-1} \mathcal{I}_{k}$ belongs to $\mathcal{I}$, hence, by definition, it is a subset of some cube from $\mathcal{K}_{i}$. This proves the second statement and, since the union of $\mathcal{K}_{0}=\mathcal{Q}_{0}$ is $\mathbf{R}^{d}$, also the last one. 
We are now ready to start our main estimates. Note that, according to Lemma 17,

$$
\varphi_{x}\left(\mathbf{R}^{d}\right)=\sum_{I \in \mathcal{I}} \varphi_{x}(G(I))
$$

and

$$
\sum_{j=i+1}^{q-1} \sum_{J \in \mathcal{I}_{j}} \varphi_{x}(G(J))=\sum_{K \in \mathcal{K}_{i+1}} \varphi_{x}(K) .
$$

Hence, using that $\left|\varphi_{x}(E)\right| \leq \psi_{x}(E)$ by Lemma 16 , we may estimate

$$
\begin{aligned}
\int & \left(\varphi_{x}\left(\mathbf{R}^{d}\right)\right)^{2} d \mu(x) \\
= & \sum_{I, J \in \mathcal{I}} \int \varphi_{x}(G(I)) \varphi_{x}(G(J)) d \mu(x) \\
= & \sum_{i=0}^{q-1} \sum_{I, J \in \mathcal{I}_{i}} \int \varphi_{x}(G(I)) \varphi_{x}(G(J)) d \mu(x) \\
& +2 \sum_{i=0}^{q-2} \sum_{j=i+1}^{q-1} \sum_{I \in \mathcal{I}_{i}} \sum_{J \in \mathcal{I}_{j}} \int \varphi_{x}(G(I)) \varphi_{x}(G(J)) d \mu(x) \\
\leq & \sum_{i=0}^{q-1} \sum_{I, J \in \mathcal{I}_{i}} \int \psi_{x}(G(I)) \psi_{x}(G(J)) d \mu(x) \\
& +2 \sum_{i=0}^{q-2} \sum_{I \in \mathcal{I}_{i}} \sum_{K \in \mathcal{K}_{i+1}} \int \varphi_{x}(G(I)) \varphi_{x}(K) d \mu(x) .
\end{aligned}
$$

For $i \geq 1$ we divide the integral $\int \psi_{x}(G(I)) \psi_{x}(G(J)) d \mu(x)$ in (13) into integrals over $\bar{Q}_{(i-1) q}(I) \cap Q_{(i-1) q}(J)$ and $\mathbf{R}^{d} \backslash\left(Q_{(i-1) q}(I) \cap Q_{(i-1) q}(J)\right)$ and, using that $\psi_{x}$ is a non-negative measure, estimate the second integral by twice the integral over $\mathbf{R}^{d} \backslash Q_{(i-1) q}(I)$. Similarly, we divide the integral $\int \varphi_{x}(G(I)) \varphi_{x}(K) d \mu(x)$ in (14) into integrals over $\mathbf{R}^{d} \backslash K$ and $K$ and replace $\varphi_{x}$ by $\psi_{x}$ in the first, but then estimate the second integral in a slightly more sophisticated way by

$$
\begin{aligned}
\int_{K} \varphi_{x}(G(I)) \varphi_{x}(K) d \mu(x) \\
=\int_{K}\left(\varphi_{x}(G(I))-\varphi_{\zeta(K)}(G(I))\right) \varphi_{x}(K) d \mu(x) \\
\quad+\int_{K} \varphi_{\zeta(K)}(G(I)) \varphi_{x}(K) d \mu(x) \\
\leq \sup _{z \in K}\left|\varphi_{z}(G(I))-\varphi_{\zeta(K)}(G(I))\right| \int_{K} \psi_{x}(K) d \mu(x) \\
\quad+\varphi_{\zeta(K)}(G(I)) \int_{K} \varphi_{x}(K) d \mu(x)
\end{aligned}
$$

where $\zeta(K)$ denotes the centre of $K$. Thus 


$$
\begin{aligned}
& \int\left(\varphi_{x}\left(\mathbf{R}^{d}\right)\right)^{2} d \mu(x) \\
& \leq \sum_{I, J \in \mathcal{I}_{0}} \int \psi_{x}(G(I)) \psi_{x}(G(J)) d \mu(x) \\
&+\sum_{i=1}^{q-1} \sum_{I, J \in \mathcal{I}_{i}} \int_{Q_{(i-1) q}(I) \cap Q_{(i-1) q}(J)} \psi_{x}(G(I)) \psi_{x}(G(J)) d \mu(x) \\
&+2 \sum_{i=1}^{q-1} \sum_{I, J \in \mathcal{I}_{i}} \int_{\mathbf{R}^{d} \backslash Q_{(i-1) q}(I)} \psi_{x}(G(I)) \psi_{x}(G(J)) d \mu(x) \\
&+2 \sum_{i=0}^{q-2} \sum_{I \in \mathcal{I}_{i}} \sum_{K \in \mathcal{K}_{i+1}} \int_{\mathbf{R}^{d} \backslash K} \psi_{x}(G(I)) \psi_{x}(K) d \mu(x) \\
&+2 \sum_{i=0}^{q-2} \sum_{I \in \mathcal{I}_{i}} \sum_{K \in \mathcal{K}_{i+1}} \sup _{z \in K}\left|\varphi_{z}(G(I))-\varphi_{\zeta(K)}(G(I))\right| \int_{K} \psi_{x}(K) d \mu(x)(1) \\
&+2 \sum_{i=0}^{q-2} \sum_{I \in \mathcal{I}_{i}} \sum_{K \in \mathcal{K}_{i+1}} \varphi_{\zeta(K)}(G(I)) \int_{K} \varphi_{x}(K) d \mu(x)
\end{aligned}
$$

Taking into account Lemma 14, we conclude that to finish the proof of Lemma 12 it suffices to estimate each of the terms (15-20) by $\mathcal{C} n^{3 / 2} \mu\left(\mathbf{R}^{d}\right)$, or by $\mathcal{C} q^{3} \mu\left(\mathbf{R}^{d}\right)$, since $n \leq q^{2} \leq(\sqrt{n}+1)^{2} \leq 4 n$ implies $n^{3 / 2} \leq q^{3} \leq 8 n^{3 / 2}$. This will be done, term by term, in a series of lemmas. The first of them follows immediately from the definitions.

Lemma 18. If $0 \leq i<j \leq n$, then

$$
\psi_{x}\left(Q_{i}(x) \backslash Q_{j}(x)\right)=\sum_{k=i}^{j-1} \omega_{k}(x) \leq j-i .
$$

In particular,

$$
\psi_{x}\left(\mathbf{R}^{d}\right)=\psi_{x}\left(Q_{0}(x) \backslash Q_{n}(x)\right) \leq n \leq q^{2} .
$$

Lemma 19. Whenever $0 \leq l<m \leq n$ and $x \in I \in \mathcal{Q}_{l}$, then

$$
\psi_{x}\left(Q_{m}(x) \cap G(I)\right)=0 .
$$

Proof. Since $\psi_{x}\left(Q_{m}(x) \cap G(I)\right) \leq \sum_{k=m}^{n-1} \omega_{k}(x)$, the statement is obvious if $m=$ $n$ or $\omega_{k}(x)=0$ for all $m \leq k \leq n-1$. On the other hand, if $p \geq m$ is the least index for which $\omega_{p}(x) \neq 0$, then $Q_{p}(z)=Q_{p}(x) \in \mathcal{I}$ for all $z \in Q_{p}(x)$, so $k(z) \geq p$ for all such $z$ and we infer that $Q_{p}(x) \cap G(I)=\emptyset$. Hence $\psi_{x}\left(Q_{m}(x) \cap G(I)\right) \leq$ $\sum_{k=m}^{p-1} \omega_{k}(x)=0$, since $\omega_{k}(x)=0$ for all $m \leq k<p$.

Lemma 20. If $0 \leq l \leq i<j \leq n$ and $\mathcal{J} \subseteq \mathcal{I} \cap \bigcup_{k=i}^{j-1} \mathcal{Q}_{k}$, then

$$
\sum_{I \in \mathcal{J}, x \in Q_{l}(I)} \psi_{x}(G(I)) \leq 2(j-l) .
$$


Consequently,

$$
\sum_{I, J \in \mathcal{J}} \int_{Q_{l}(I) \cap Q_{l}(J)} \psi_{x}(G(I)) \psi_{x}(G(J)) d \mu(x) \leq 4(j-l)^{2} \mu\left(\mathbf{R}^{d}\right) .
$$

Proof. If $I \in \mathcal{J}$ and $x \in Q_{l}(I) \backslash I$, then $G(I) \subseteq I \subseteq Q_{l}(x) \backslash Q_{j}(x)$. Using also the disjointness of $G(I)(I \in \mathcal{I})$ (Lemma 17) and Lemma 18, we have

$$
\sum_{I \in \mathcal{J}, x \in Q_{l}(I) \backslash I} \psi_{x}(G(I)) \leq \psi_{x}\left(Q_{l}(x) \backslash Q_{j}(x)\right) \leq j-l .
$$

Similarly, but using first Lemma 19, we get

$$
\sum_{I \in \mathcal{J}, x \in I} \psi_{x}(G(I))=\sum_{I \in \mathcal{J}, x \in I} \psi_{x}\left(G(I) \backslash Q_{j}(x)\right) \leq \psi_{x}\left(Q_{l}(x) \backslash Q_{j}(x)\right) \leq j-l .
$$

Adding these two inequalities gives the first statement. The second statement follows, since

$$
\begin{aligned}
& \sum_{I, J \in \mathcal{J}} \int_{Q_{l}(I) \cap Q_{l}(J)} \psi_{x}(G(I)) \psi_{x}(G(J)) d \mu(x) \\
& =\int\left(\sum_{I \in \mathcal{J}, x \in Q_{l}(I)} \psi_{x}(G(I))\right)^{2} d \mu(x) \leq 4(j-l)^{2} \mu\left(\mathbf{R}^{d}\right) .
\end{aligned}
$$

Estimate of (15). From Lemma 20 with $\mathcal{J}=\mathcal{I}_{0}$ we infer

$$
\sum_{I, J \in \mathcal{I}_{0}} \int \psi_{x}(G(I)) \psi_{x}(G(J)) d \mu(x) \leq 4 q^{3} \mu\left(\mathbf{R}^{d}\right) .
$$

Estimate of (16). Using Lemma 20, we see that

$$
\begin{gathered}
\sum_{i=1}^{q-1} \sum_{I, J \in \mathcal{I}_{i}} \int_{Q_{(i-1) q}(I) \cap Q_{(i-1) q}(J)} \psi_{x}(G(I)) \psi_{x}(G(J)) d \mu(x) \\
\leq \sum_{i=0}^{q-1} 4(2 q)^{2} \mu\left(\mathbf{R}^{d}\right)=16 q^{3} \mu\left(\mathbf{R}^{d}\right) .
\end{gathered}
$$

Lemma 21. If $0 \leq l<k \leq n$ and $I \in \mathcal{I} \cap \mathcal{Q}_{k}$, then

$$
\int_{Q_{l}(I) \backslash Q_{l+1}(I)} \psi_{x}(I) d \mu(x) \leq 2 C_{1} C_{2} 2^{(l-k)(d-\alpha)} \mu(I) / \sigma .
$$

Proof. We first note that $\mu(I) \geq 2^{-k \alpha} C_{2}^{-1} \sigma$ since $k>0$ and $I \in \mathcal{I} \cap \mathcal{Q}_{k}$. If $x \in$ $Q_{l}(I) \backslash Q_{l+1}(I)$ then $I \cap\left(Q_{i}(x) \backslash \bar{Q}_{i+1}(x)\right)=\emptyset$ if $i \neq l$ and $I \cap\left(Q_{l}(x) \backslash Q_{l+1}(x)\right)=I$. Hence

$$
\begin{aligned}
\psi_{x}(I) & =\mathcal{L}^{d}(I)\left(2^{-l d}-2^{-(l+1) d}\right)^{-1} \omega_{l}(x) \leq 2^{-k d} 2^{l d+1} \omega_{l}(x) \\
& \leq 2^{-k(d-\alpha)} 2^{l d+1} C_{2} \mu(I) \omega_{l}(x) / \sigma
\end{aligned}
$$


Since, according to Lemma 15,

$$
\int_{Q_{l}(I) \backslash Q_{l+1}(I)} \omega_{l}(x) d \mu(x) \leq C_{1} 2^{-l \alpha}
$$

we infer that

$$
\begin{aligned}
& \int_{Q_{l}(I) \backslash Q_{l+1}(I)} \psi_{x}(I) d \mu(x) \\
& \quad \leq 22^{-k(d-\alpha)} 2^{l d+1} C_{2} \mu(I) \int_{Q_{l}(I) \backslash Q_{l+1}(I)} \omega_{l}(x) d \mu(x) / \sigma \\
& \quad \leq 2 C_{1} C_{2} 2^{(l-k)(d-\alpha)} \mu(I) / \sigma .
\end{aligned}
$$

Lemma 22. If $0 \leq m \leq k \leq n$ and $I \in \mathcal{I} \cap \mathcal{Q}_{k}$, then

$$
\int_{\mathbf{R}^{d} \backslash Q_{m}(I)} \psi_{x}(I) d \mu(x) \leq 2 C_{1} C_{2} 2^{(m-k)(d-\alpha)} \mu(I) /\left(\sigma\left(2^{d-\alpha}-1\right)\right) .
$$

In particular,

$$
\int_{\mathbf{R}^{d} \backslash I} \psi_{x}(I) d \mu(x) \leq 2 C_{1} C_{2} \mu(I) /\left(\sigma\left(2^{d-\alpha}-1\right)\right) .
$$

Proof. Since $\psi_{x}\left(\mathbf{R}^{d} \backslash Q_{0}(x)\right)=0$, the first inequality follows by adding the inequalities from Lemma 21 for $l=0,1, \ldots, m-1$. The second inequality is the case $m=k$ of the first one.

Estimate of (17). Using that $\sum_{J \in \mathcal{I}_{i}} \psi_{x}(G(J)) \leq n \leq q^{2}$ according to Lemma 18 and that according to Lemma 22 , for any $I \in \overline{\mathcal{I}}_{i}$,

$$
\int_{\mathbf{R}^{d} \backslash Q_{(i-1) q}(I)} \psi_{x}(G(I)) d \mu(x) \leq \mathcal{C}_{1} 2^{-q(d-\alpha)} \mu(I),
$$

where $\mathcal{C}_{1}=2 C_{1} C_{2} /\left(\sigma\left(2^{d-\alpha}-1\right)\right)$, we estimate that

$$
\begin{aligned}
& \sum_{i=1}^{q-1} \sum_{I, J \in \mathcal{I}_{i}} \int_{\mathbf{R}^{d} \backslash Q_{(i-1) q}(I)} \psi_{x}(G(I)) \psi_{x}(G(J)) d \mu(x) \\
& \quad \leq q^{2} \sum_{i=1}^{q-1} \sum_{I \in \mathcal{I}_{i}} \int_{\mathbf{R}^{d} \backslash Q_{(i-1) q}(I)} \psi_{x}(G(I)) d \mu(x) \\
& \quad \leq \mathcal{C}_{1} 2^{-q(d-\alpha)} q^{2} \sum_{i=1}^{q-1} \sum_{I \in \mathcal{I}_{i}} \mu(I) \\
& \leq \mathcal{C}_{1} 2^{-q(d-\alpha)} q^{4} \mu\left(\mathbf{R}^{d}\right) \\
& \leq \mathcal{C}_{2} q^{3} \mu\left(\mathbf{R}^{d}\right)
\end{aligned}
$$

where $\mathcal{C}_{2}=\mathcal{C}_{1} \sup _{m \geq 1} 2^{-m(d-\alpha)} m$. 
Estimate of (18). We first note that the disjointness of the cubes from $\mathcal{K}_{i+1}$ and the second inequality of Lemma 22 imply that

$$
\sum_{K \in \mathcal{K}_{i+1}} \int_{\mathbf{R}^{d} \backslash K} \psi_{x}(K) d \mu(x) \leq \mathcal{C}_{1} \sum_{K \in \mathcal{K}_{i+1}} \mu(K) \leq \mathcal{C}_{1} \mu\left(\mathbf{R}^{d}\right) .
$$

Thus, using first that $\sum_{I \in \mathcal{I}_{i}} \psi_{x}(G(I)) \leq q^{2}$, we have

$$
\begin{aligned}
& \sum_{i=0}^{q-2} \sum_{I \in \mathcal{I}_{i}} \sum_{K \in \mathcal{K}_{i+1}} \int_{\mathbf{R}^{d} \backslash K} \psi_{x}(G(I)) \psi_{x}(K) d \mu(x) \\
& \leq q^{2} \sum_{i=0}^{q-2} \sum_{K \in \mathcal{K}_{i+1}} \int_{\mathbf{R}^{d} \backslash K} \psi_{x}(K) d \mu(x) \\
& \leq q^{2} \sum_{i=0}^{q-2} \mathcal{C}_{1} \mu\left(\mathbf{R}^{d}\right) \\
& \leq \mathcal{C}_{1} q^{3} \mu\left(\mathbf{R}^{d}\right) .
\end{aligned}
$$

Lemma 23. For any dyadic cube $K$,

$$
\sum_{I \in \mathcal{I}} \sup _{x, y \in K}\left|\varphi_{x}(G(I) \backslash K)-\varphi_{y}(G(I) \backslash K)\right| \leq 1 .
$$

Proof. If $x, y \in K \in \mathcal{Q}_{m}$ and $0 \leq k<m$, then $Q_{k}(x)=Q_{k}(y), Q_{k+1}(x)=$ $Q_{k+1}(y)$ and $\omega_{k}(x)=\omega_{k}(y)$. Using also that $\omega_{k}(x) 2^{k \alpha} \mu\left(Q_{k}(x)\right) \leq C_{1}$ by Lemma 15 and that, for all $u \in Q_{k}(x),\left|H_{k}(y, u)-H_{k}(x, u)\right| \leq C_{2} 2^{k \alpha+k-m}$ by assumption, we infer that

$$
\begin{aligned}
\left|H_{k}(y)-H_{k}(x)\right| & \leq \omega_{k}(x) \int_{Q_{k}(x)}\left|H_{k}(y, u)-H_{k}(x, u)\right| d \mu(u) \\
& \leq C_{2} \omega_{k}(x) 2^{k \alpha} \mu\left(Q_{k}(x)\right) 2^{k-m} \\
& \leq C_{1} C_{2} 2^{k-m} .
\end{aligned}
$$

Hence

$$
\begin{aligned}
& \left|\varphi_{x}(G(I) \backslash K)-\varphi_{y}(G(I) \backslash K)\right| \\
& \quad \leq\left(C_{1} C_{2}\right)^{-1} \sum_{k=0}^{m-1} \frac{\mathcal{L}^{d}\left(G(I) \cap\left(Q_{k}(x) \backslash Q_{k+1}(x)\right)\right)}{\mathcal{L}^{d}\left(Q_{k}(x) \backslash Q_{k+1}(x)\right)} C_{1} C_{2} 2^{k-m}
\end{aligned}
$$

and, taking supremum over $x, y \in K$, we have

$$
\begin{aligned}
& \sup _{x, y \in K}\left|\varphi_{x}(G(I) \backslash K)-\varphi_{y}(G(I) \backslash K)\right| \\
& \leq \sum_{k=0}^{m-1} \frac{\mathcal{L}^{d}\left(G(I) \cap\left(Q_{k}(\zeta(K)) \backslash Q_{k+1}(\zeta(K))\right)\right)}{\mathcal{L}^{d}\left(Q_{k}(\zeta(K)) \backslash Q_{k+1}(\zeta(K))\right)} 2^{k-m} .
\end{aligned}
$$

Thus, summing over $I \in \mathcal{I}$ and using the disjointness of the sets $G(I)$, we get 


$$
\sum_{I \in \mathcal{I}} \sup _{x, y \in K}\left|\varphi_{x}(G(I) \backslash K)-\varphi_{y}(G(I) \backslash K)\right| \leq \sum_{k=0}^{m-1} 2^{k-m} \leq 1 .
$$

Estimate of (19). To use Lemma 23, we observe that, whenever $u \in K \in \mathcal{K}_{i+1}$ and $I \in \bigcup_{j=0}^{i} \mathcal{I}_{j}$, then $k(u)>i$ and hence $u \notin G(I)$. Consequently, $\varphi_{x}(G(I))=$ $\varphi_{x}(G(I) \backslash K)$ for every $x \in K$ and, using first that $\int_{K} \psi_{x}(K) d \mu(x) \leq q^{2} \mu(K)$, since $\psi_{x}(K) \leq \psi_{x}\left(\mathbf{R}^{d}\right) \leq q^{2}$ by Lemma 18 , then Lemma 23 and finally the disjointness of $\mathcal{K}_{i+1}$, we infer that

$$
\begin{aligned}
& \sum_{i=0}^{q-2} \sum_{I \in \mathcal{I}_{i}} \sum_{K \in \mathcal{K}_{i+1}} \sup _{z \in K}\left|\varphi_{z}(G(I))-\varphi_{\zeta(K)}(G(I))\right| \int_{K} \psi_{x}(K) d \mu(x) \\
& \leq q^{2} \sum_{i=0}^{q-2} \sum_{K \in \mathcal{K}_{i+1}} \mu(K) \sum_{j=0}^{i} \sum_{I \in \mathcal{I}_{j}} \sup _{z \in K}\left|\varphi_{z}(G(I))-\varphi_{\zeta(K)}(G(I))\right| \\
& \quad=q^{2} \sum_{i=0}^{q-2} \sum_{K \in \mathcal{K}_{i+1}} \mu(K) \sum_{j=0}^{i} \sum_{I \in \mathcal{I}_{j}} \sup _{z \in K}\left|\varphi_{z}(G(I) \backslash K)-\varphi_{\zeta(K)}(G(I) \backslash K)\right| \\
& \quad \leq q^{2} \sum_{i=0}^{q-2} \sum_{K \in \mathcal{K}_{i+1}} \mu(K) \\
& \leq q^{3} \mu\left(\mathbf{R}^{d}\right) .
\end{aligned}
$$

Lemma 24. For every dyadic cube $K$,

$$
\int_{K} \varphi_{x}(K) d \mu(x)=0 .
$$

Proof. If $Q \in \mathcal{Q}_{k}$, the anti-symmetry of $H_{k}$ and the fact that $\omega_{k}(x)=\omega_{k}(\zeta(Q))$ for every $x \in Q$ imply that

$$
\int_{Q} H_{k}(x) d \mu(x)=\omega_{k}(\zeta(Q)) \int_{Q} \int_{Q} H_{k}(x, u) d \mu(x) d \mu(u)=0 .
$$

Hence, if $K \in \mathcal{Q}_{m}$,

$$
\begin{aligned}
\int_{K} \varphi_{x}(K) d \mu(x) & =\left(C_{1} C_{2}\right)^{-1} \sum_{k=m}^{n-1} \int_{K} H_{k}(x) d \mu(x) \\
& =\left(C_{1} C_{2}\right)^{-1} \sum_{k=m}^{n-1} \sum_{Q \in \mathcal{Q}_{k}, Q \subseteq K} \int_{Q} H_{k}(x) d \mu(x) \\
& =0 .
\end{aligned}
$$

Estimate of (20). By Lemma 24,

$$
\sum_{i=0}^{q-2} \sum_{I \in \mathcal{I}_{i}} \sum_{K \in \mathcal{K}_{i+1}} \varphi_{\zeta(K)}(G(I)) \int_{K} \varphi_{x}(K) d \mu(x)=0 .
$$




\section{Proof of the Palm formula}

Noting that trivial cases have been handled in Proposition 2, we assume, for the rest of this section, that $0<\alpha<d$ and a measure $\mu \in \mathcal{M}\left(\mathbf{R}^{d}\right)$ are fixed.

We will need to make some, not very difficult but careful, estimates of measures of sets. This will be facilitated by defining the usual distances $F_{k}(\nu, \tilde{\nu})$ of measures $\nu$ and $\tilde{\nu}$ as the supremum of $\left|\int f(w) d \nu(w)-\int f(w) d \tilde{\nu}(w)\right|$, where $f$ runs through functions $f: \mathbf{R}^{d} \rightarrow \mathbf{R}$ having support in $B(0, k)$ and Lipschitz constant at most one. It is not difficult to see that $\left(F_{k}\right)_{k=1}^{\infty}$ is a sequence of pseudo-metrics inducing the weak topology of $\mathcal{M}\left(\mathbf{R}^{d}\right)$ (see e.g. [Mat, 14.13]). In addition, we will only need the following simple estimate of the distance of enlargements of measures.

Lemma 25. Whenever $x, \tilde{x} \in \mathbf{R}^{d}$ and $r>0$, then

$$
F_{k}\left(\frac{\mu_{x, r}}{r^{\alpha}}, \frac{\mu_{\tilde{x}, r}}{r^{\alpha}}\right) \leq 2 \frac{\mu(B(x,\|x-\tilde{x}\|+k r))}{r^{\alpha}} \frac{\|x-\tilde{x}\|}{r} .
$$

Proof. If $f$ is as in the definition of $F_{k}$ then

$$
\begin{aligned}
& \left|\int f(w) d \mu_{x, r}(w)-\int f(w) d \mu_{\tilde{x}, r}(w)\right| \\
& \quad=\int\left|f\left(\frac{w-x}{r}\right)-f\left(\frac{w-\tilde{x}}{r}\right)\right| d \mu(w) \\
& \quad \leq \int_{B(x, k r)} \frac{\|x-\tilde{x}\|}{r} d \mu(w)+\int_{B(\tilde{x}, k r)} \frac{\|x-\tilde{x}\|}{r} d \mu(w) \\
& \quad \leq 2 \mu(B(x,\|x-\tilde{x}\|+k r)) \frac{\|x-\tilde{x}\|}{r},
\end{aligned}
$$

which, divided by $r^{\alpha}$, proves the statement.

Before coming to our last lemma, we need to introduce the distances also in the space $\mathcal{M}\left(\mathbf{R}^{d}\right) \times \mathbf{R}^{d}$. We define them by $\varrho_{p}((\nu, u),(\tilde{\nu}, \tilde{u}))=F_{p}(\nu, \tilde{\nu})+\|u-\tilde{u}\|$; they form a non-decreasing sequence of pseudo-metrics generating the topology of $\mathcal{M}\left(\mathbf{R}^{d}\right) \times \mathbf{R}^{d}$. We will also use the usual notation $\varrho_{p}((\nu, u), M)=$ $\inf _{(\tilde{\nu}, \tilde{u}) \in M} \varrho_{p}((\nu, u),(\tilde{\nu}, \tilde{u}))$ for the $\varrho_{p}$ distance of $(\nu, u) \in \mathcal{M}\left(\mathbf{R}^{d}\right) \times \mathbf{R}^{d}$ from a set $M \subseteq \mathcal{M}\left(\mathbf{R}^{d}\right) \times \mathbf{R}^{d}$ and recall that the $\varrho_{p}$ distance of sets $M, N \subseteq \mathcal{M}\left(\mathbf{R}^{d}\right) \times \mathbf{R}^{d}$ is defined as the infimum of $\varrho_{p}((\nu, u),(\tilde{\nu}, \tilde{u}))$, where $(\nu, u) \in M$ and $(\tilde{\nu}, \tilde{u}) \in N$.

Lemma 26. Suppose that $R>1$ and that $U \subseteq V \subseteq \mathcal{M}\left(\mathbf{R}^{d}\right) \times B(0, R-1)$ are open subsets of $\mathcal{M}\left(\mathbf{R}^{d}\right) \times \mathbf{R}$ such that, for some positive integer $p$, the $\varrho_{p}$ distance of $U$ from the complement of $V$ is positive and the values of $\nu(B(0,5 R+p+1))$ for $(\nu, u) \in U$ are bounded. Then, for $\mu$-almost every $x \in \mathbf{R}^{d}$,

$$
\liminf _{\delta \downarrow 0} \iint\left[1_{V}(\nu, y)-1_{U}\left(T^{y} \nu,-y\right)\right] d \nu(y) d P_{\delta}^{x}(\nu) \geq 0 .
$$

Proof. We choose $\xi \in(0,1)$ such that $\varrho_{p}((\nu, u),(\tilde{\nu}, \tilde{u}))>\xi$ whenever $(\nu, u) \in U$ and $(\tilde{\nu}, \tilde{u}) \notin V$, and we let 


$$
A_{1}=1+\sup _{(\nu, u) \in U} \nu(B(0,5 R+p+1)), \quad A_{2}=\left(2 A_{1}+1\right) / \xi
$$

and observe that our assumptions imply that $A_{1}$ is finite. (It may be pointed out that the names of the constants are chosen with the future application of Lemma 11 in mind. Also, to avoid trivialities, note that in the case $U=\emptyset$ the statement of the lemma is obvious.)

Define $S: \mathbf{R}^{d} \times \mathbf{R}^{d} \times(0, \infty) \rightarrow \mathcal{M}\left(\mathbf{R}^{d}\right) \times \mathbf{R}^{d}$ by

$$
S(x, y ; r)=\left(\frac{\mu_{x, r}}{r^{\alpha}}, \frac{y-x}{r}\right) .
$$

Since $S$ is continuous, the set $S^{-1}(U)$ is open, which implies that the function

$$
H(x, y ; r)=\max \left\{0,1-A_{2} \inf _{(\tilde{x}, \tilde{y} ; r) \in S^{-1}(U)} \frac{\|x-\tilde{x}\|+\|y-\tilde{y}\|}{r}\right\} .
$$

is upper semi-continuous, hence Borel measurable.

We prove that

$$
H(x, y ; r) \leq 1_{V}\left(\frac{\mu_{x, r}}{r^{\alpha}}, \frac{y-x}{r}\right)
$$

for all $(x, y ; r) \in \mathbf{R}^{d} \times \mathbf{R}^{d} \times(0, \infty)$. Indeed, otherwise there would exist $(x, y ; r)$ and $(\tilde{x}, \tilde{y} ; r)$ such that $S(x, y ; r) \notin V, S(\tilde{x}, \tilde{y} ; r) \in U$, and $\|x-\tilde{x}\|+\|y-\tilde{y}\|<r / A_{2}$, and we would infer from $S(\tilde{x}, \tilde{y} ; r) \in U$ that

$$
\begin{aligned}
\mu(B(\tilde{x},\|\tilde{x}-x\|+p r)) & \leq \mu(B(\tilde{x},(p+1) r))=\mu_{\tilde{x}, r}(B(0,(p+1))) \\
& \leq \mu_{\tilde{x}, r}(B(0,5 R+p+1))<A_{1} r^{\alpha} .
\end{aligned}
$$

But then Lemma 25 would imply that

$$
\begin{aligned}
\xi & <\varrho_{p}\left(\left(\frac{\mu_{x, r}}{r^{\alpha}}, \frac{y-x}{r}\right),\left(\frac{\mu_{\tilde{x}, r}}{r^{\alpha}}, \frac{\tilde{y}-\tilde{x}}{r}\right)\right) \\
& \leq 2 \frac{\mu(B(\tilde{x},\|\tilde{x}-x\|+p r))}{r^{\alpha}} \frac{\|x-\tilde{x}\|}{r}+\frac{\|x-\tilde{x}\|+\|y-\tilde{y}\|}{r} \\
& \leq 2 A_{1} / A_{2}+1 / A_{2} \\
& =\xi .
\end{aligned}
$$

Noting that, for any $G$,

$$
\begin{aligned}
\iint G(\nu, y) d \nu(y) d P_{\delta}^{x}(\nu) & =\frac{1}{|\log \delta|} \int_{\delta}^{1} \int G\left(\frac{\mu_{x, r}}{r^{\alpha}}, y\right) \frac{d \mu_{x, r}(y)}{r^{\alpha}} \frac{d r}{r} \\
& =\frac{1}{|\log \delta|} \int_{\delta}^{1} \int G\left(\frac{\mu_{x, r}}{r^{\alpha}}, \frac{y-x}{r}\right) d \mu(y) \frac{d r}{r^{1+\alpha}}
\end{aligned}
$$

we integrate (21) first with respect to $d \mu(y)$ and then with respect to $d r / r^{1+\alpha}$ to find out that

$$
\frac{1}{|\log \delta|} \int_{\delta}^{1} \int H(x, y ; r) d \mu(y) \frac{d r}{r^{1+\alpha}} \leq \iint 1_{V}(\nu, y) d \nu(y) d P_{\delta}^{x}(\nu)
$$


for every $x \in \mathbf{R}^{d}$ and every $\delta>0$. In a similar way we find an estimate of the integral involving the set $U$ : Since $H(y, x ; r)=1$ if $S(y, x ; r) \in U$, we have

$$
1_{U}\left(\frac{\mu_{y, r}}{r^{\alpha}}, \frac{x-y}{r}\right) \leq H(y, x ; r) .
$$

Hence, noting that, for any $G$,

$$
\begin{aligned}
\iint G\left(T^{y} \nu,-y\right) d \nu(y) d P_{\delta}^{x}(\nu) & =\frac{1}{|\log \delta|} \int_{\delta}^{1} \int G\left(\frac{T^{y} \mu_{x, r}}{r^{\alpha}},-y\right) \frac{d \mu_{x, r}(y)}{r^{\alpha}} \frac{d r}{r} \\
& =\frac{1}{|\log \delta|} \int_{\delta}^{1} \int G\left(\frac{\mu_{y, r}}{r^{\alpha}}, \frac{x-y}{r}\right) d \mu(y) \frac{d r}{r^{1+\alpha}}
\end{aligned}
$$

we obtain, integrating (23) first with respect to $d \mu(y)$ and then with respect to $d r / r^{1+\alpha}$, that

$$
\iint 1_{U}\left(T^{y} \nu,-y\right) d \nu(y) d P_{\delta}^{x}(\nu) \leq \frac{1}{|\log \delta|} \int_{\delta}^{1} \int H(y, x ; r) d \mu(y) \frac{d r}{r^{1+\alpha}}
$$

for every $x \in \mathbf{R}^{d}$ and every $\delta>0$.

We prove that $H(x, y ; r)$ satisfies the assumptions of Lemma 11 . We have already noted that $H$ is Borel measurable, and it is clear that its range is contained in $[0,1]$. If $H(x, y ; r) \neq 0$, then we find $(\tilde{x}, \tilde{y} ; r) \in S^{-1}(U)$ such that $\|x-\tilde{x}\|+$ $\|y-\tilde{y}\| \leq r / A_{2} \leq r$ and infer from $S(\tilde{x}, \tilde{y} ; r) \in U$ that $\|\tilde{x}-\tilde{y}\|<(R-1) r$ and $\mu_{\tilde{x}, r}(B(0,5 R+1))<A_{1} r^{\alpha}$. Hence

$$
\|x-y\| \leq\|x-\tilde{x}\|+\|y-\tilde{y}\|+\|\tilde{x}-\tilde{y}\|<r+(R-1) r=R r,
$$

which proves 11(i), and

$$
\mu(B(x, 5 R r)) \leq \mu(B(\tilde{x},(5 R+1) r))=\mu_{\tilde{x}, r}(B(0,5 R+1))<A_{1} r^{\alpha},
$$

which proves 11(ii). Finally, the requirement 11(iii) follows directly from the definition of $H(x, y ; r)$.

This finishes the proof of the Lemma, since (24) and (22) and Lemma 11 imply that

$$
\begin{aligned}
& \liminf _{\delta \downarrow 0} \iint\left[1_{V}(\nu, y)-1_{U}\left(T^{y} \nu,-y\right)\right] d \nu(y) d P_{\delta}^{x}(\nu) \\
& \quad \geq \liminf _{\delta \rightarrow 0} \frac{1}{|\log \delta|} \int_{\delta}^{1} \int \frac{H(x, y ; r)-H(y, x ; r)}{r^{\alpha}} d \mu(y) \frac{d r}{r} \\
& \quad=0 .
\end{aligned}
$$

Proof of Theorem 1

Using that $\mathcal{M}\left(\mathbf{R}^{d}\right) \times \mathbf{R}^{d}$ is a separable metric space, we find a countable basis $\mathcal{U}$ of its open sets which is closed under finite unions. Let $\tilde{\mathcal{U}}$ be the set of pairs $(U, V)$ of sets from $\mathcal{U}$ for which there are $R>1$ and a positive integer $p$ such that $U \subseteq V \subseteq \mathcal{M}\left(\mathbf{R}^{d}\right) \times B(0, R-1)$, the $\varrho_{p}$ distance of $U$ from the complement of $V$ is positive and the values of $\nu(B(0,5 R+p+1))$ for $(\nu, u) \in U$ are bounded. 
Since $\tilde{\mathcal{U}}$ is countable, we infer from Lemma 26 that there is a $\mu$ null set $N \subseteq \mathbf{R}^{d}$ such that for every $x \in \mathbf{R}^{d} \backslash N$,

$$
\liminf _{\delta \downarrow 0} \iint\left[1_{V}(\nu, y)-1_{U}\left(T^{y} \nu,-y\right)\right] d \nu(y) d P_{\delta}^{x}(\nu) \geq 0
$$

for every pair $(U, V) \in \tilde{\mathcal{U}}$.

Let $x \in \mathbf{R}^{d} \backslash N$ and $P \in \mathcal{P}^{\alpha}(\mu, x)$ be fixed. Let $\delta_{k} \downarrow 0$ be such that $P_{\delta_{k}}^{x}$ converge weakly to $P$. Then

$$
\Lambda_{k}(E)=\iint 1_{E}(\nu, y) d \nu(y) d P_{\delta_{k}}^{x}(\nu)
$$

are, according to Lemma 7 , Radon measures on $\mathcal{M}\left(\mathbf{R}^{d}\right) \times \mathbf{R}^{d}$ converging vaguely to the Radon measure

$$
\Lambda(E)=\iint 1_{E}(\nu, y) d \nu(y) d P(\nu) .
$$

Moreover, since the mapping $\tilde{T}: \mathcal{M}\left(\mathbf{R}^{d}\right) \times \mathbf{R}^{d} \rightarrow \mathcal{M}\left(\mathbf{R}^{d}\right) \times \mathbf{R}^{d}$ defined by $\tilde{T}(\nu, y)=\left(T^{y} \nu,-y\right)$ is a homeomorphism, the measures $\tilde{\Lambda}_{k}=\Lambda_{k} \circ \tilde{T}^{-1}$ converge vaguely to $\tilde{\Lambda}=\Lambda \circ \tilde{T}^{-1}$.

We intend to use Lemma 5 to show that $\tilde{\Lambda}<\Lambda$. Whenever $K \subseteq L$ are subsets of $\mathcal{M}\left(\mathbf{R}^{d}\right) \times \mathbf{R}^{d}$ such that $K$ is compact and $L$ is open, we first choose $R>2$ such that $K \subseteq \mathcal{M}\left(\mathbf{R}^{d}\right) \times B(0, R-2)$ and find $V \in \mathcal{U}$ such that $K \subseteq V \subseteq$ $L \cap \mathcal{M}\left(\mathbf{R}^{d}\right) \times B(0, R-1)$. Then we choose a positive integer $p$ such that the $\varrho_{p}$ distance of $K$ from the complement of $V$ is positive. We let $\xi$ denote this distance, define

$$
A=1+\sup _{(\nu, u) \in K} \nu(B(0,5 R+p+1))
$$

and

$$
\hat{U}=\left\{(\nu, u) \in V: \varrho_{p}((\nu, u), K)<\xi / 2, \nu(B(0,5 R+p+1))<A\right\},
$$

and choose $U \in \mathcal{U}$ such that $K \subseteq U \subseteq \hat{U}$. Then $(U, V) \in \tilde{\mathcal{U}}$, and we conclude from (25) that

$$
\begin{aligned}
\liminf _{k \rightarrow \infty} \tilde{\Lambda}_{k}(U) & =\liminf _{k \rightarrow \infty} \iint 1_{U}\left(T^{y} \nu,-y\right) d \nu(y) d P_{\delta_{k}}^{x}(\nu) \\
& \leq \liminf _{k \rightarrow \infty} \iint 1_{V}(\nu, y) d \nu(y) d P_{\delta_{k}}^{x}(\nu) \\
& =\liminf _{k \rightarrow \infty} \Lambda_{k}(V) \\
& \leq \limsup _{k \rightarrow \infty} \Lambda_{k}(L) .
\end{aligned}
$$

By Lemma 5 , this shows that $\tilde{\Lambda} \leq \Lambda$. But then $\Lambda=\tilde{\Lambda} \circ \tilde{T}^{-1} \leq \Lambda \circ \tilde{T}^{-1}=\tilde{\Lambda}$, and we conclude that $\tilde{\Lambda}=\Lambda$, which is the statement of Theorem 1 . 


\section{References}

[APa] M. Arbeiter and N. Patzschke. Random self-similar multifractals. Math. Nachr., $181: 5-42,1996$.

[Ba] C. Bandt. The tangent distribution for self similar measures. Lecture at the 5th Conference on Real Analysis and Measure Theory, Capri, 1992.

[BeFi] T. Bedford and A.M. Fisher. Analogues of the Lebesgue density theorem for fractal sets of reals and integers. Proc. London Math. Soc.(3), 64:95-124, 1992.

[FaSp] K.J. Falconer and O.B. Springer. Order-two density of sets and measures with nonintegral dimension. Mathematika, 42:1-14, 1995.

[Fe] H. Federer. Geometric Measure Theory. Springer Verlag, Berlin, 1968.

[G] S. Graf. On Bandt's tangential distribution for self similar measures. Mh. Math., 120:223-246, 1995.

[K] O. Kallenberg. Random Measures. Akademie-Verlag, Berlin, 1983.

[Ma1] J.M. Marstrand. The $(\phi, s)$-regular subsets of $n$-space. Trans. Am. Math. Soc., 113:369-392, 1964.

[Ma2] J.M. Marstrand. Order-two density and the strong law of large numbers. Mathematika, 43:1-22, 1996 .

[Mat] P. Mattila. The Geometry of Sets and Measures in Euclidean Spaces. Cambridge University Press, Cambridge, 1995.

[Me] J. Mecke. Stationäre zufällige Maße auf lokalkompakten abelschen Gruppen. Zeitschr. Wahrsch. verw. Gebiete, 9:36-58, 1967.

[Mö1] P. Mörters. Tangent Measure Distributions and the Geometry of Measures. PhD thesis, University College London, 1995.

[Mö2] P. Mörters. Symmetry properties of average densities and tangent measure distributions of measures on the line. To appear in Adv. Math., 1996.

[MöPr] P. Mörters and D. Preiss. On one-sided average densities of fractal measures on the line. To appear in Sem. d'initiation à l'analyse, Publ. Univ. Pierre et Marie Curie, Paris, 1996.

[O1] T. C. O'Neil. A Local Version of the Projection Theorem and Other Results in Geometric Measure Theory. PhD Thesis, University College London, 1995.

[O2] T. C. O'Neil. A local version of the projection theorem. Proc. London Math. Soc.(3), 73:68-104, 1996.

[Pr] D. Preiss. Geometry of measures in $\mathbf{R}^{n}$ : Distribution, rectifiability and densities. Ann. Math., 125:537-643, 1987.

[PaZU] N. Patzschke and U. Zähle. Self-similar random measures IV. The recursive construction model of Falconer, Graf, Mauldin and Williams. Math. Nachr., 149:285-302, 1990 .

[PaZM] N. Patzschke and M. Zähle. Self-similar random measures are locally scale invariant. Prob. Theory Rel. Fields, 97:559-574, 1993.

[S] L. Schwartz. Radon measures on arbitrary topological spaces and cylindrical measures. Oxford University Press, 1973.

[T] F. Topsøe. Topology and Measure. Springer LNM 133, 1970.

[V] M. Valadier. Young measures. In: Methods of Nonconvex Analysis, Springer LNM 1446, pp.152-188, 1989.

[ZU] U. Zähle. Self-similar random measures I. Notion, carrying Hausdorff dimension and hyperbolic distribution. Prob. Theory Rel. Fields, 80:79-100, 1988. 
This article was processed by the author using the $\mathrm{LAT}_{\mathrm{E}} \mathrm{X}$ style file cljour1 from Springer-Verlag. 